\title{
Short intervals almost all containing primes
}

\author{
by \\ N. WATt (Nottingham)
}

1. Introduction. This paper is about the infimum $\left(\theta_{0}\right)$ of those constants $\theta$ for which almost all of the intervals $\left[n, n+n^{\theta}\right]$ contain primes (here "almost all" signifies that there can only be $o(N)$ exceptions with integer $n \leq N)$. It is not known if $\theta_{0}=0$, but by 1943 Selberg [31] had shown that $\theta_{0} \leq 19 / 77$. In the same paper he proved that if the Riemann Hypothesis is true, then almost all of the intervals $\left[n, n+f(n)(\log n)^{2}\right]$ will contain a prime; provided only that $f(n) \rightarrow \infty$. Heath-Brown [11] has reduced the power of the logarithm to 1 , (the limit) subject to an additional assumption about the vertical distribution of the zeros of $\zeta(s)$.

It has been shown unconditionally by Motohashi [28] that, for $\varepsilon>0$, almost all the intervals $\left[n, n+n^{\varepsilon}\right]$ contain numbers with exactly two prime factors. And this has seen successive improvements by Wolke [35], by Harman [7] and, most recently, by Mikawa [24], so that now one can put $f(n)(\log n)^{5}$ in place of $n^{\varepsilon}$ (provided $f(n) \rightarrow \infty$ ).

Building upon a method of Halász, Montgomery obtained new results on the distribution of zeros of $\zeta(s)$. With these he proved that $\theta_{0} \leq 1 / 5$ and that Hoheisel's asymptotic formula,

$$
\pi(x)-\pi(x-y) \sim \frac{y}{\log x}
$$

(where $\pi(x)$ is the number of primes $p \leq x$ ), is valid with $y=x^{\theta}$, for any fixed $\theta>3 / 5$ (see [25], Chapter 14). Huxley [14] reduced $3 / 5$ to $7 / 12$ through an improvement of the Halász-Montgomery method. His zero density estimate yields $\theta_{0} \leq 1 / 6$.

For (1.1), the exponent $7 / 12$ has yet to be improved upon, but Iwaniec and Jutila [17] have combined a linear sieve with the analytic methods in an argument that gives

$$
\pi(x)-\pi(x-y) \gg y / \log x
$$

with $y=x^{\theta}$, for any fixed $\theta>5 / 9$. This was reduced to $11 / 20$ by HeathBrown and Iwaniec [13], and further progress was made by authors whom we 
shall mention later. Then Harman [8] used a linear sieve to prove $\theta_{0} \leq 1 / 10$. In unpublished work, which was reported in [9], he has improved this to $\theta_{0} \leq 1 / 12$ through an unconventional approach (developed in [9] for a different problem) to the application of the linear sieve. An independent proof (also unpublished, but sketched in [12]) was found at around the same time by Heath-Brown. In his forthcoming paper [19] Jia Chaohua has got $\theta_{0} \leq 1 / 13$. Li Hongze has a manuscript [21] improving this to $\theta_{0} \leq 2 / 27$.

In this paper we aim to show that $\theta_{0} \leq 1 / 14$. To this end we shall consider the integer intervals $\mathcal{A}=(x-y, x] \cap \mathbb{Z}$ with $X / 2<x<X$ and $y / x=Y / X$, where $X$ and $Y$ are given and $X^{\theta}<Y<X / 2$. We find it convenient to write

$$
f(x, y)=\bar{O}(F),
$$

when $y=y(x)$ is understood and

$$
\left(\frac{1}{X} \int_{X / 2}^{X}|f(x, y(x))|^{2} d x\right)^{1 / 2}=O(F) .
$$

Note that, by a Minkowski inequality,

$$
\bar{O}\left(F_{1}\right)+\ldots+\bar{O}\left(F_{r}\right)=\bar{O}\left(F_{1}+\ldots+F_{r}\right),
$$

where the implicit constant on the right is the maximum of those on the left. The bound $\theta_{0} \leq 1 / 14$ is an immediate consequence of the following result:

Theorem 1. If $E \geq 1$ and $Y \geq X^{1 / 14}(\log X)^{3 E+13}$, then

$$
\pi(x)-\pi(x-y) \geq \frac{c y}{\log X}+\bar{O}_{E}\left(Y(\log X)^{-E}\right),
$$

where $c$ is an absolute positive constant and the conventions are those of the preceding paragraph.

Our calculations, the results of which are summarised in Section 6, show that $c=1 / 6$ is admissible in Theorem 1 . This is not the optimal value obtainable by the methods employed here.

In the course of revising this paper the author received a preprint from Li Hongze and another [20] (due to appear soon) from Jia Chaohua: both about $\theta_{0}$. Jia can show $\theta_{0} \leq 1 / 14$. His method differs from ours in several respects, and it is remarkable that it succeeds with only the fourth power moment of the Riemann zeta-function where we have used our Theorem 2 (below). The new work [22] of Li Hongze has $\theta_{0} \leq 1 / 15$. In it several results of this paper get used (Proposition 3.1, Lemma 5.1 and Lemma $5.3)$.

Montgomery and Vaughan [27] have observed that results like Theorem 1 can help to show that a short interval contains lots of Goldbach numbers 
(even numbers representable as a sum of two primes). Indeed, if (1.2) holds with $y=x^{\theta}$ for some fixed $\theta>0$, if $\alpha$ is a constant such that Theorem 1 remains true with $\alpha$ in place of the exponent $1 / 14$, and if $\pi_{2}(x)$ denotes the number of Goldbach numbers not exceeding $x$, then

$$
\pi_{2}(x)-\pi_{2}(x-y) \gg y,
$$

with $y=x^{\beta}$, for any fixed $\beta>\theta \alpha$. We are grateful to Professors J. Pintz and A. Perelli, from whom we learned of this. As they pointed out in the introduction to [30], the proof is essentially Ramachandra's. For deeper results about Goldbach numbers in short intervals see [30], [19] and [20].

The proof of Theorem 1 occupies the remaining sections of this paper. Note that the theorem is trivial for $X=O(1)$, so that whenever it becomes desirable that $X \geq C$ (some constant) we will be able to assume that this is indeed the case. The value of $X$ may remain the same throughout, but alternative values of $Y$ need consideration at several junctures, and it is only at the conclusion that $Y>X^{1 / 14}$ will be a necessary assumption.

Two new ingredients facilitate the improvement over $\theta_{0} \leq 1 / 10$, the last result with a published proof [8]. The first ingredient, from Harman's paper [9], is a way of using linear sieve results as part of a procedure to obtain useful formulae:

$$
\sum_{m} a_{m} S\left(\mathcal{A}_{m}, X^{\phi}\right)=(1+O(\delta)) y \lambda(x)+\bar{O}_{\delta, j}\left(Y(\log X)^{-j}\right)
$$

where $\left(a_{m}\right)$ is a suitable sequence of non-negative real numbers, $\mathcal{A}_{m}=\{n \in$ $\mathcal{A}: n \equiv 0(\bmod m)\}, S\left(\mathcal{A}_{m}, z\right)=\#\left\{n \in \mathcal{A}_{m}: n \notin \mathcal{A}_{p}\right.$, for all primes $p<z\}$ and $\lambda(x)$ does not depend on $Y$. Harman employs the (elementary) Buchstab identity,

$$
\sum_{m} a_{m} S\left(\mathcal{A}_{m}, X^{\phi}\right)=\sum_{m} a_{m} S\left(\mathcal{A}_{m}, X^{\delta}\right)-\sum_{m} a_{m} \sum_{X^{\delta} \leq p<X^{\phi}} S\left(\mathcal{A}_{m p}, p\right)
$$

which is valid for $0 \leq \delta \leq \phi$ when $\left(a_{m}\right)$ is a sequence with $a_{m}=0$ for values of $m$ that are divisible by at least one prime $p<X^{\phi}$ (in which case we refer to $\left(a_{m}\right)$ as an $X^{\phi}$-sifted sequence). We utilise (1.6) in a much less subtle way than Harman does in [9], where (1.6) is applied arbitrarily many times in an induction argument (see [10] for an analysis of the method). Here the motivating problem is different and just a few applications of (1.6) suffice.

The other new ingredient is the following mean-value result from the author's paper [34]: 
Theorem 2. Let $\left|b_{n}\right| \leq 1$ for $n=1,2, \ldots$ Then, for $T \geq N^{4}$ and $\varepsilon>0$,

$$
I(T, N)=\frac{1}{T} \int_{0}^{T}\left|\sum_{n=1}^{N} b_{n} n^{i t}\right|^{2}\left|\zeta\left(\frac{1}{2}+i t\right)\right|^{4} d t \ll_{\varepsilon} T^{\varepsilon} N .
$$

For the estimation of the $\bar{O}$-term in (1.5) we rely exclusively on meansquare bounds for Dirichlet polynomials (see Section 2); just such a bound (Proposition 2.2) is an easy corollary of Theorem 2. For $\delta$ sufficiently small, Proposition 2.2 allows one to obtain formulae like (1.5) for the single sum on the right of $(1.6)$ subject to constraints on $\left(a_{m}\right)$ which are weaker than the corresponding constraints in, for example, [8].

The proof of Theorem 2 followed the work [4], [5] of Deshouillers and Iwaniec. In [5] those authors found a different bound for $I(T, N)$ which implies the case $T \geq N^{5}$ of Theorem 2 , and can give better results for sequences $\left(b_{n}\right)$ that are in some sense sparser than those we need consider here. Their result was used by Iwaniec and Pintz [18] to prove (1.2), with $y=x^{\theta}$, for $\theta>\frac{11}{20}-\frac{1}{406}$. This was reduced to $\frac{11}{20}-\frac{1}{386}$ by Mozzochi [29], and then quite substantially to $\frac{11}{20}-\frac{1}{220}=0.5454 \ldots$, by Lou and Yao [23]. Very recently Baker and Harman have announced that they can improve this to $\theta \geq 0.535$. Their manuscript [1] introduces new combinatorial techniques to the problem, including the fundamental idea from [9] which we use here. Although we expect that Theorem 2 would make at most a small improvement to these figures (see [29]), it has a more worthwhile effect when bounds for $\theta_{0}$ are sought.

Theorem 2 can be applied quite directly when $\delta=0$ and $S\left(\mathcal{A}_{m}, X^{\delta}\right)=$ $\# \mathcal{A}_{m}$, subject only to quite simple conditions on the sequence $\left(a_{m}\right)$. Other factors, however, demand that $\delta>0$, so some sieving is required. We use the linear Rosser-Iwaniec sieve (Lemma 3.1) and control the remainder terms using Theorem 2. Usually the sieving would complicate the conditions on $\left(a_{m}\right)$, but it is a nice feature of the Harman approach that, by taking the sieving level to be $D=X^{\Delta}$ with $\Delta^{2}=\delta$, and $\Delta>0$ (for example), one can ensure that as $\delta \rightarrow 0$ the conditions on $\left(a_{m}\right)$ relax and converge towards those at $\delta=0$. At the end of Section 6 it is shown that, for a sufficiently small fixed value of $\delta$, the whole proof of Theorem 1 goes through to a successful conclusion.

No sieving is necessary in order to get a formula like (1.5) for the double sum in (1.6). Exceptionally bad behaviour is ruled out, because

$$
\sum_{p} p^{i t} \ll_{A, B} P \log ^{-A} P \quad\left(\log ^{A} P<t<P^{B}\right)
$$

when $A$ and $B$ are arbitrary positive constants and $p$ runs over the primes in any subinterval of an interval $[P / 2, P]$. This is a corollary of results of 
Vinogradov about $\zeta(s)$ and $\zeta^{\prime}(s) / \zeta(s)$ with $s=\sigma+i t$ near $\sigma=1$ (see, for example, [8], p. 346). After (1.7), Harman's application (Lemma 2.6) of Huxley's Halász Lemma (Lemma 2.5) is enough. For $Y=X^{\theta}$ with $\theta<$ $1 / 2$ we certainly need (1.7) with $t$ as large as $X^{1-\theta}>X^{1 / 2}$. Hence $t<$ $P^{B}$ implies $P>X^{1 /(2 B)}$. This is the origin of the requirement that $\delta>0$. Lemma 2.5, which determined Huxley's exponent $7 / 12$, also determines (via Proposition 2.1) the maximum permissible value of $\phi$ here: for $\theta=1 / 14$ it is $\phi=12 / 77$.

To apply the formulae (1.5) we appeal to the Buchstab identity (1.6). This gives (as in [12], (7)),

$$
\begin{aligned}
S & =S\left(\mathcal{A}, X^{1 / 2}\right)=\pi(x)-\pi(x-y) \\
& =S\left(\mathcal{A}, X^{\phi}\right)-\sum_{X^{\phi} \leq p<X^{1 / 2}} S\left(\mathcal{A}_{p}, p\right) \\
& =S\left(\mathcal{A}, X^{\phi}\right)-\sum_{X^{\phi} \leq p<X^{1 / 2}} S\left(\mathcal{A}_{p}, X^{\phi}\right)+\sum_{X^{\phi} \leq q<p<X^{1 / 2}} \sum_{\substack{\text { s. }\\
}} S\left(\mathcal{A}_{p q}, q\right) \\
& =\Sigma_{1}-\Sigma_{2}+R \quad \text { (say) }
\end{aligned}
$$

where $p$ and $q$ run over the primes. Formulae like (1.5) can be obtained for $\Sigma_{1}$ and $\Sigma_{2}$ here, even without recourse to Theorem 2 (see the remark after Proposition 2.2). Using the prime number theorem in the form

$$
\pi(x)=\int_{2}^{x} \frac{d u}{\log u}+O_{A}\left(x \log ^{-A} x\right),
$$

where $A$ is an arbitrary positive constant (see [3], Chapter 18), one can show that in the formulae for $\Sigma_{1}$ and $\Sigma_{2}$ the corresponding functions $\lambda_{1}(x)$ and $\lambda_{2}(x)$ (say) may be replaced by terms $c_{1} / \log X$ and $c_{2} / \log X$ with explicit positive constants $c_{1}, c_{2}$.

Note that in order to make use of (1.9) we take $Y=X \log ^{-B} X$, for some constant $B$. Nevertheless, the functions $c_{i} / \log X$ and $\lambda_{i}(x)$ are independent of $Y$, so that if the formula for $\Sigma_{i}$ is valid for all $Y$ in some set containing $X \log ^{-B} X$ then the corresponding formula obtained by replacing $\lambda_{i}(x)$ with $c_{i} / \log X$ will hold for the same set of $Y$ values. The general principle involved here is used with abandon throughout this paper (see, for example, the proofs of Lemmas 2.3, 3.4, 5.1 and 5.4).

Inconveniently $c_{2}>c_{1}$ when $\theta=1 / 14$ and $\phi=12 / 77$ (see Section 4 ), but Theorem 2, through the important Proposition 3.1, allows formulae similar to (1.5) to be found for two marginally modified parts of the remaining sum $R$ in (1.8) (see Lemmas 5.1 and 5.3). Lemma 5.5 can handle certain other parts: those where a Hölder inequality involving three factors is particularly effective. The rest of $R$ ( $R-R^{\prime}$, say) is trivially non-negative; and we are able to prove Theorem 1 with $c=c_{1}-c_{2}+c^{\prime}+O(\delta)>0$, where $R^{\prime} \geq$ 
$\left(c^{\prime}+O(\delta)\right) y / \log X$ for almost all of the sets $\mathcal{A}$.

D. R. Heath-Brown has pointed out that the exponent $1 / 14$ in Theorem 1 is not especially significant and can certainly be improved to some extent at the cost of reducing the constant $c$ (see [22]). Indeed, the optimal constant $c$ obtainable by elaboration of the arguments laid out in this paper must be a continuous function of $\theta$. The discontinuities (with respect to $\theta$ ) of the hypotheses for Proposition 2.1 do not contradict this. But to replace the condition $Y \geq X^{1 / 14}(\log X)^{3 E+13}$ by any condition of the shape $Y \geq X^{\theta}$ with $\theta \leq 1 / 14$ would considerably complicate the proof of Theorem 1 .

The author is indebted to Glyn Harman for his original suggestion that Theorem 2 might be used to improve the upper bound on $\theta_{0}$, and for several enlightening discussions that aided the completion of this work. He would also like to thank D. R. Heath-Brown for communicating details of his unpublished work. Thanks are due to the referee for helping to improve the clarity of some sections. Some results mentioned in the introduction were made known to the author by Professors R. C. Baker, J. B. Friedlander and A. Schinzel.

The research was largely completed while the author was a research associate at the University of Wales College of Cardiff. It was written up into a paper while the author resided at Göttingen as a guest of the Sonderforschungsbereich 170 "Geometrie und Analysis". The author is grateful to Frau Christina Gieseking for typing the manuscript.

Notation. Greek and Roman letters in the usual typeface denote complex numbers or functions. The letters $d, h, k, l, m, n, r$ always denote positive integers. The letters $p$ and $q$ always denote primes.

The integer and fractional parts of $x \in \mathbb{R}$ are written as $[x]$ and $\{x\}$ (although we sometimes use $\{x\}$ to denote a set containing one element, $x$ ). The divisor function $\tau_{r}(n)$ is the coefficient of $n^{-s}$ in the Dirichlet series for $\zeta^{r}(s)$. We write $\mu(d)$ for the Möbius function and $(m, n)$ for the greatest common divisor of $m$ and $n$. If $\mathcal{B}$ is a set, then $\# \mathcal{B}$ denotes the cardinality of $\mathcal{B}$.

As usual $O(F)$ denotes a term $z$ satisfying $|z| \leq c F$, where $c$ is a positive constant. The notation $f \ll g$ means the same as $f=O(g)$, and the notation $f \asymp g$ is short for: $f \ll g \ll f$. In all these notations, variables upon which the implicit constants necessarily depend will appear as suffixes (to $O$, «, or $\asymp)$. This practice extends to the $\bar{O}$-notation defined by (1.3).

The functions $\xi(u)$ and $\omega(s)$ have particular meanings. The former is the characteristic function of the interval $(x-y, x]$. The latter is the Buchstab function defined in Lemma 4.1 and extended so that $\omega(s)=0$ for $s<1$.

All other unusual conventions and notations have already been explained 
in the introduction. In particular we note: the conventions, governing $X, Y$, $x, y$ and $\mathcal{A}$, that were brought in before Theorem 1 , and the definitions of $\mathcal{A}_{m}$ and $S\left(\mathcal{A}_{m}, z\right)$. The $z$-sifted sequences, which crop up quite often, were defined under (1.6).

2. Dirichlet polynomials. In this section we present some lemmas which show how results of the form

$$
\sum_{x-y<h \leq x} \eta_{h}=y \lambda(x)+\bar{O}\left(\frac{Y}{\log ^{j} X}\right)
$$

depend on mean square estimates for Dirichlet polynomials

$$
H(t)=\sum_{1 \leq h \leq C X} \eta_{h} h^{i t},
$$

where $C$ is a constant and $C \geq 1$. The relevant estimates are given at the end of the section. They include the only new result in this section, Proposition 2.2.

The sequences $\left(\eta_{h}\right)$ that we consider will satisfy

$$
\left|\eta_{h}\right| \leq \tau_{r}(h)
$$

for $h=1,2, \ldots$, with some fixed integer $r \geq 1$.

Lemma 2.1. For $r, l \geq 1$ and $\theta \geq \varepsilon>0$,

$$
\sum_{X-X^{\theta}<h \leq X} \tau_{r}^{l}(h) \ll_{r, l, \varepsilon} X^{\theta}(\log X)^{r^{l}-1} .
$$

In particular, for $r \geq 1, \varepsilon>0$ and $h \ll X, \tau_{r}(h) \ll_{r, \varepsilon} X^{\varepsilon}$.

Proof. The first bound is a result of Shiu [32], Theorem 2. The second bound is a corollary of the first.

Lemma 2.2. Let $H(t)$ and $\left(\eta_{h}\right)$ be as in (2.2) and (2.3). Suppose that $2 \leq T_{0} \leq T \leq X^{1-\varepsilon}$. Then, for $X / 2<x<X$ and $0<y<x / 2$,

$$
\frac{1}{y} \sum_{x-y<h \leq x} \eta_{h}=\lambda(x)+f(x, y)+O_{r, \varepsilon}\left(\left(\frac{X \log T}{y T}+\frac{T_{0}^{2} y}{X}\right)(\log X)^{r-1}\right),
$$

where

$$
\lambda(x)=\frac{T_{0}}{\pi x} \sum_{1 \leq h \leq C X} \eta_{h} \frac{\sin \left(T_{0} \log (x / h)\right)}{T_{0} \log (x / h)}
$$

and

$$
f(x, y)=\frac{1}{2 \pi i}\left\{\int_{-T}^{-T_{0}}+\int_{T_{0}}^{T}\right\}\left(\frac{x^{i t}-(x-y)^{i t}}{y}\right) H(-t) \frac{d t}{t} .
$$


Proof. This is a straightforward application of the formula

$$
\frac{1}{2 \pi i} \int_{-T}^{T}\left(\varrho^{i t}-1\right) \frac{d t}{t}=O\left(\frac{1}{T|\log \varrho|}\right)+ \begin{cases}1 / 2 & \text { if } \varrho>1, \\ -1 / 2 & \text { if } 1 / \varrho>1\end{cases}
$$

(from [12]), with $\varrho=x / h$ or $\varrho=(x-y) / h$. Note that with $\varrho=x / h$, for example, one can do better in the range $|h-x|<x / T \leq x / 2$, where the above integral is bounded by

$$
\int_{-T}^{T}|t \log \varrho| \frac{d t}{|t|} \ll T\left|\log \frac{x}{h}\right| \ll 1 .
$$

The remaining $h$ fall into $O(\log T)$ ranges of the form $H / 2<|h-x| \leq H$, with $X \gg H \gg X / T \geq X^{\varepsilon}$. Hence Lemma 2.1 applies and the first of the $O$-terms comes out. The second arises when the integral over the range $-T_{0} \leq t \leq T_{0}$ is simplified using

$$
\frac{x^{i t}-(x-y)^{i t}}{i t}-y x^{i t-1}=-\int_{x-y}^{x} \int_{u}^{x}(i t-1) v^{i t-2} d v d u \ll \frac{y^{2}}{x^{2}}|t+i|
$$

and, from Lemma 2.1 again, $H(t) \ll_{r} X(\log X)^{r-1}$.

LEMMA 2.3. Under the same conditions as Lemma 2.2, and with $K, L \geq$ $1, K L=X, K / K_{1}=L_{1} / L=C$, we have

$$
\begin{aligned}
& \frac{1}{y}\left|\sum_{K_{1}<k \leq K} \eta_{k}\left(\left[\frac{x}{k}\right]-\left[\frac{x-y}{k}\right]-\frac{y}{k}\right)\right| \\
& \leq\left|f^{*}(x, y)\right|+\left|f^{*}(x, z)\right|+O_{r, \varepsilon}\left(\left(\frac{X \log T}{y T}+\frac{T_{0}}{(L \log X)^{1 / 2}}\right)(\log X)^{r}\right),
\end{aligned}
$$

where $z=x / T_{0}(L \log X)^{1 / 2}$ and $f^{*}(x, y)$ is $f(x, y)$ with $H(t)$ replaced by

$$
H^{*}(t)=\sum_{K_{1}<k \leq K} \eta_{k} k^{i t} \sum_{L / 4<l \leq L_{1}} l^{i t}=\sum_{h=1}^{\infty} \eta_{h}^{*} h^{i t},
$$

say (so that, in place of (2.3), one has $\left|\eta_{h}^{*}\right| \leq \tau_{r+1}(h)$ ).

Proof. This approximates a result from Heath-Brown and Iwaniec [13], Section 4 . We prove it here by first observing

$$
g(y)=\sum_{K_{1}<k \leq K} \eta_{k}\left(\left\{\frac{x-y}{k}\right\}-\left\{\frac{x}{k}\right\}\right) \ll_{r} K(\log X)^{r-1}
$$

(see Lemma 2.1). Hence the lemma is trivial unless $y<z$, which we henceforth assume. The sum to be bounded is

$$
\frac{g(y)}{y}=\frac{1}{y} \sum_{x-y<h \leq x} \eta_{h}^{*}-\sum_{K_{1}<k \leq K} \frac{\eta_{k}}{k} .
$$


And, as $L \geq 1$, we have $0<z / x<1 / 2$. Therefore Lemma 2.2 applies to give

$$
\frac{g(y)}{y}-\frac{g(z)}{z}=f^{*}(x, y)-f^{*}(x, z)+O_{r, \varepsilon}\left(\left(\frac{X \log T}{y T}+\frac{T_{0}^{2} z}{X}\right)(\log X)^{r}\right),
$$

and the lemma follows by (2.4) with $y=z$.

The next lemma shows that the term $f(x, y)$ from Lemma 2.2 will be $\bar{O}\left(\log ^{-j} X\right)$, consistent with $(2.1)$, if

$$
\int_{T_{0}}^{T}|H(t)|^{2} d t \ll X^{2}(\log X)^{-2 j} ;
$$

and hence that the same can be said of $f^{*}(x, y)$ and $f^{*}(x, z)$ in Lemma 2.3, subject to an identical condition on $H^{*}(t)$.

LEMma 2.4. Assuming the same hypotheses and conventions as Lemma 2.2 ,

$$
\int_{X / 2}^{X}|f(x, \varrho x)|^{2} d x \ll \frac{1}{X} \int_{T_{0}}^{T}|H(t)|^{2} d t,
$$

uniformly for $0<\varrho \leq 1 / 2$.

Pr o o f. See Harman [8], Lemma 2. A logarithm can be saved by working with

$$
\int_{1}^{2} \int_{X /(2 \beta)}^{\beta X}|f(x, \varrho x)|^{2} d x d \beta .
$$

The classical mean value theorem for Dirichlet polynomials is:

$$
\int_{0}^{T}\left|\sum_{h=1}^{H} \eta_{h} h^{i t}\right|^{2} d t=(T+O(H)) \sum_{h=1}^{H}\left|\eta_{h}\right|^{2}
$$

(see [26]). For $T \ll X$ (and $H \asymp X$ ) the bounds (2.6), (2.3) and Lemma 2.1 give (2.5) with $j=\left(1-r^{2}\right) / 2 \leq 0$, which just falls short of being useful to us. We end this section with some results supplying that little extra which is required for a non-trivial formula (2.1).

We shall assume that $H(t)$ can be written as a suitable product of Dirichlet polynomials:

$$
M(t)=\sum_{m} a_{m} m^{i t}, \quad N(t)=\sum_{n} b_{n} n^{i t}, \quad L(t)=\sum_{l} c_{l} l^{i t},
$$

where $L, M, N \geq 1, a_{m}=0$ for $m>M, b_{n}=0$ for $n>N$, and $c_{l}=0$ for $l>L$. Certainly some assumption is necessary as (2.6) is best possible in 
the general case. For $t \in \mathbb{R}$ the Cauchy-Schwarz inequality yields,

$$
|M(t)|^{2 h} \leq M^{h} \sum_{r}\left|\sum_{m_{1} m_{2} \ldots m_{h}=r} \ldots a_{m_{1}} a_{m_{2}} \ldots a_{m_{h}}\right|^{2}=M^{h} G_{h},
$$

say. Huxley [14], Section 2 has obtained the following "large-values" result:

Lemma 2.5 (Halász-Montgomery-Huxley). Suppose that, for $r=1,2, \ldots$ $\ldots, R$, we have $\left|M\left(t_{r}\right)\right| \geq V>0$, with $t_{r} \in \mathbb{R}$. Suppose also that $1 \leq$ $\left|t_{r}-t_{s}\right| \leq 2 T$ for $1 \leq r<s \leq R$. Then for $h=1,2, \ldots$,

$$
R \ll G_{h} M^{h} V^{-2 h}+G_{h}^{3} M^{h} T V^{-6 h} \log ^{2}\left(4 M^{h} T\right) .
$$

Using this and the classical mean-value theorem (2.6) Harman [8], Lemma 4 could get the following useful lemma:

Lemma 2.6 (Harman). Let $T \geq 2$ and $2 h \leq \beta<6 h$, where $h$ is an integer. Let $\mathfrak{m}$ be a measurable subset of the interval $[-T, T]$, and suppose that $M(t)$ from (2.7) satisfies $|M(t) / M|^{\beta-2 h} \leq \mu(t \in \mathfrak{m})$. Then

$$
\int_{\mathfrak{m}}|M(t)|^{\beta} d t \ll\left(1+\frac{T / \mu}{M^{h} \log \left(T M^{h}\right)}\left(\frac{\lambda^{2} T / \mu}{M^{2 h}}\right)^{\frac{\beta-2 h}{6 h-\beta}}\right) M^{\beta} \mu \lambda,
$$

where $\lambda=G_{h} M^{-h} \log \left(T M^{h}\right)$ with $G_{h}$ as in (2.8).

For bounded sequences $\left(a_{m}\right)$, Lemma 2.1 could be used to estimate $G_{h}$ in (2.8). In dealing with $X^{\delta}$-sifted sequences, however, we make use of the following observation: if $\left(a_{m}^{(1)}\right), \ldots,\left(a_{m}^{(h)}\right)$ are $X^{\delta}$-sifted sequences of numbers whose absolute values do not exceed unity, then for $1 \leq r \leq X^{C}$,

$$
\sum_{m_{1} \ldots m_{h}=r} \ldots a_{m_{1}}^{(1)} \ldots a_{m_{h}}^{(h)} \ll_{h, \delta, C} 1
$$

Hence, when Lemma 2.6 applies to such a sequence $\left(a_{m}\right)$ and

$$
M(t) \ll_{\delta} M(\log M)^{-A} \quad(t \in \mathfrak{m})
$$

with some $A \geq 0$, then it will show that

$$
\int_{\mathfrak{m}}|M(t)|^{\beta} d t \ll_{\delta, h} M^{\beta}(\log M)^{1-(\beta-2 h) A}
$$

provided that

$$
M^{\beta+2 h} \geq T^{4}(\log M)^{4(\beta-2 h)(A+1)} .
$$

Proposition 2.1. Let $X=M N, M \geq X^{\delta} \geq 2$ and

$$
X^{1-\theta}(\log X)^{-2 A-1}=T \geq 2
$$

with $A \geq 0$ and $1 /(2 k+2) \leq \theta<1 /(2 k)$ for some integer $k \geq 4$. Let $M(t)$ and $N(t)$ be Dirichlet polynomials of the form given in (2.7), where the sequences $\left(a_{m}\right)$ and $\left(b_{n}\right)$ are now both $X^{\delta}$-sifted and satisfy $\left|a_{m}\right| \leq 1$, 
$\left|b_{n}\right| \leq 1$ for all $m$ and $n$. Suppose further that $M(t)$ satisfies (2.10) with $A$ as above and with $\mathfrak{m}$ a measurable subset of the interval $[-T, T]$. Then

$$
\int_{\mathfrak{m}}|M N(t)|^{2} d t \ll_{\delta, A} X^{2}(\log X)^{1-2 A},
$$

provided that

$$
M \leq X^{\theta+\frac{1-\theta}{2 k-1}}(\log X)^{-A} .
$$

Proof. In essence we follow the argument of Harman in [8], Lemma 5. Note first that the upper bound on $M$ implies that $N \geq T^{1-1 /(2 k-1)} \geq T^{6 / 7}$. If $N \geq T$, then by Lemma 2.6 (with $\beta=2 h=2$ and $\mu=1$ ) we have

$$
\int_{\mathfrak{M}}|N(t)|^{2} d t \ll N^{2} \log N,
$$

where $\mathfrak{M}$ can be taken to be the whole interval $[-T, T]$. This, together with (2.10), suffices to prove the proposition in the case $N \geq T$. Similarly, in proving the proposition in the case $N \leq T$ one may take, in $\mathfrak{m}$ 's stead, any measurable set $\mathfrak{m}_{1} \subset \mathfrak{m}$ for which $\mathfrak{M}=\mathfrak{m} \backslash \mathfrak{m}_{1}$ satisfies (2.13). We shall use

$$
\mathfrak{m}_{1}=\left\{t \in \mathfrak{m}:|N(t)| \leq T^{1 / 4} N^{1 / 2} \log N\right\} .
$$

The corresponding integral in (2.13) with $\mathfrak{M}=\mathfrak{m} \backslash \mathfrak{m}_{1}$, is bounded above by the supremum of all possible sums of $|N(t)|^{2}$ taken over $t=t_{1}, \ldots, t_{R} \in \mathfrak{M}$ with $\left|t_{r}-t_{s}\right| \geq 1$ for $r \neq s$. Such a sum $S$ can be split into $O(\log N)$ sums $S\left(V_{1}\right), S\left(V_{2}\right), \ldots$ by dividing the range for $|N(t)|$ into intervals $\left[V_{j} / 2, V_{j}\right]$ with $T^{1 / 4} N^{1 / 2} \log N \leq V_{j} \leq 2 N$. Then, by Lemma 2.5 ,

$$
S\left(V_{j}\right) \ll N^{2}+N^{4} T V_{j}^{-4} \log ^{2} N
$$

and

$$
S \ll N^{2} \log N+N^{4} T\left(T^{1 / 4} N^{1 / 2} \log N\right)^{-4} \log ^{2} N,
$$

showing, as required, that $\mathfrak{M}$ satisfies (2.13).

Given an integer $h \geq k$ and a real number $\alpha$ with $0 \leq \alpha \leq 1$, we use Hölder's inequality to bound the integral over $\mathfrak{m}_{1}$, in terms of

$$
\int_{\mathfrak{m}_{1}}|M(t)|^{2(h+\alpha)} d t \text { and } \int_{\mathfrak{m}_{1}}|N(t)|^{2 \gamma} d t,
$$

where $1 /(h+\alpha)+1 / \gamma=1$. For $t \in \mathfrak{m}_{1}$, we have

$$
|N(t)| \leq T^{1 / 4} N^{1 / 2} \log N \leq N^{19 / 24} \log N \ll_{A, h} N(\log N)^{-A(h+\alpha)} .
$$

Using this bound and the trivial bound $|M(t)| \leq M$ (instead of (2.10)) we apply Lemma 2.6 to the integrals in (2.14). This results in a proof of the proposition in the case where $h \ll_{\delta} 1$,

$$
M^{2(h+\alpha)+2 h} \geq T^{4}(\log X)^{8 \alpha}
$$


and

$$
N^{2\left(1+\frac{1}{h+\alpha-1}\right)+2} \geq T^{4}(\log X)^{\frac{8(A(h+\alpha)+1)}{h+\alpha-1}}
$$

(see $(2.10)-(2.12))$.

The latter two conditions are satisfied for

$$
\begin{array}{r}
M \in\left[T^{\frac{2}{2 h+\alpha}}(\log X)^{\frac{4 \alpha}{2 h+\alpha}}, T^{\frac{\theta}{1-\theta}+\frac{1}{2 h-1+2 \alpha}}(\log X)^{\frac{2 A+1}{1-\theta}-\frac{4(A(h+\alpha)+1)}{2 h-1+2 \alpha}}\right] \\
=\left[L_{h}(\alpha), U_{h}(\alpha)\right]=\mathcal{I}_{h}(\alpha), \quad \text { say. }
\end{array}
$$

Here $L_{h}(\alpha)$ and $U_{h}(\alpha)$ are continuous functions of $\alpha \in[0,1]$. Suppose that, for $k \leq h \leq H$ (say), they are also monotonic decreasing functions of $\alpha$ with $L_{h+1}(1) \leq L_{h}(1) \leq U_{h+1}(0)$ and $L_{h}(\alpha) \leq U_{h}(\alpha)$, for $0 \leq \alpha \leq 1$. Then, for $k \leq h \leq H$, the intervals $\mathcal{I}_{h}=\left[L_{h}(1), U_{h}(0)\right]$ are covered by the intervals $\mathcal{I}_{h}(\alpha)$ with $0 \leq \alpha \leq 1$. Furthermore, consecutive intervals in the sequence $\mathcal{I}_{k}, \mathcal{I}_{k+1}, \ldots, \mathcal{I}_{H}$ overlap, so that $\mathcal{I}_{k} \cup \mathcal{I}_{k+1} \cup \ldots \cup \mathcal{I}_{H}$ is an interval $\mathcal{I}_{H}^{*}=\left[L_{H}(1), U_{k}(0)\right]$. The assumptions we have made about $L_{h}(\alpha)$ and $U_{h}(\alpha)$ can be verified by dint of a calculation (which we omit), provided that $X$ is sufficiently large in terms of $A$ and $H$. This proves the proposition for $M \in \mathcal{I}_{2 k+1}^{*}$, since we know from the inequalities $X^{\delta} \leq M<X^{1 / k}$ that $2 k+1 \ll_{\delta} 1$.

We complete the proof by showing that $L_{2 k+1}(1) \leq M \leq U_{k}(0)$. The upper bound,

$$
M \leq X^{\theta+\frac{1-\theta}{2 k-1}}(\log X)^{2 A+1-\frac{4(A k+1)+2 A+1}{2 k-1}},
$$

is slightly weaker than the bound already given in the statement of the proposition. As we are in the case $N \leq T$,

$$
M=\frac{X}{N} \geq \frac{X}{T}=X^{\theta}(\log X)^{2 A+1} .
$$

This gives the required lower bound:

$$
M \geq X^{\frac{2(1-\theta)}{4 k+3}}(\log X)^{\frac{4-2(2 A+1)}{4 k+3}} .
$$

Lemma 2.7. Let $X=M N L, M \geq X^{\varepsilon} \geq 2$ and suppose that $M(t)$, $N(t)$ and $L(t)$ are Dirichlet polynomials of the form given in (2.7), where the sequences $\left(a_{m}\right),\left(b_{n}\right)$ and $\left(c_{l}\right)$ are all $X^{\varepsilon}$-sifted and satisfy $\left|a_{m}\right| \leq 1$, $\left|b_{n}\right| \leq 1$ and $\left|c_{l}\right| \leq 1$, for all $m, n$ and $l$. Let $A \geq 0, T \geq 2$, and let $\mathfrak{m}$ be a measurable subset of $[-T, T]$ for which (2.10) holds. Finally, let $r$ be the greatest integer strictly less than $\varrho=1 /(1-1 / h-1 / k)$, where $h$ and $k$ are given integers with $2 \leq h<k$, and suppose that

$$
M \geq T^{2 /(\varrho+r)}(\log X)^{\frac{4(A+1)(\varrho-r)}{\varrho+r}}, \quad N \geq T^{1 / h} \quad \text { and } \quad L \geq T^{1 / k} .
$$

Then

$$
\int_{\mathfrak{m}}|M N L(t)|^{2} d t \ll_{\varepsilon, A, k} X^{2}(\log X)^{1-2\left(\frac{\rho-r}{\varrho}\right) A} .
$$


Proof. This is a straightforward application of Hölder's inequality followed by Lemma 2.6 (see (2.10)-(2.12)).

We shall only use the above lemma for a few small values of $h$ and $k$. But we remark that in any case, $(\varrho-r) /(\varrho+r) \leq 1 / 3$ and $(\varrho-r) / \varrho \geq 1 /(h k)$.

Lemma 2.8. Let $C>1$ and let $L(t)$ be a Dirichlet polynomial of the form given in (2.7) with coefficients $c_{l}=1$, for $L / C<l \leq L$, and $c_{l}=0$, otherwise. Then for $T \geq 2$ and $-T \leq t \leq T$,

$$
\begin{gathered}
|L(t)|^{2} \ll_{C} L(\log T) \int_{-T}^{T}\left|\zeta\left(\frac{1}{2}+i(\tau+t)\right)\right|^{2} \frac{d \tau}{|\tau+i|} \\
+\frac{L^{2}}{|t+i|^{2}}+\frac{L^{2} \log ^{2} L}{T^{2}}+1 .
\end{gathered}
$$

Proof. We follow Harman [8], formula (20) in applying the Perron formula [33], Lemma 3.12 and in shifting the contour of integration. Where he uses the bound $|\zeta(\sigma+i t)| \ll t^{1 / 6}$, we use a result,

$$
\inf _{T / 2<t<T} \int_{1 / 2}^{2}|\zeta(\sigma+i t)| d \sigma \ll \log T,
$$

which was observed in a similar connection by Iwaniec and Jutila [17] and is a corollary of [33], Theorem 7.2(A).

Proposition 2.2. Let $X=M N L$. Let $M(t), N(t)$ and $L(t)$ be Dirichlet polynomials of the form given in (2.7), where now $\left|a_{m}\right| \leq 1,\left|b_{n}\right| \leq 1$, for all $m$ and $n$, and the coefficients $c_{l}$ are as in Lemma 2.8. Let

$$
2 \leq X^{\varepsilon} \leq T_{0}<T \leq X^{1-\varepsilon}
$$

and suppose also that

$$
N^{4} \leq T \leq X^{-\varepsilon} L^{2} N
$$

Then

$$
\int_{T_{0}}^{T}|M N L(t)|^{2} d t \ll_{C, \varepsilon} X^{2-\varepsilon / 3} .
$$

Pr o of. By Lemma 2.8 the last integral is $O_{C}(H)$, where

$$
H=Z L(\log T)^{2}+P L^{2}+Q L^{2}(\log L)^{2} T^{-2}+Q
$$

with

$$
Z=\sup _{-T \leq \tau \leq T} \int_{T_{0}}^{T}\left|\zeta\left(\frac{1}{2}+i(t+\tau)\right)\right|^{2}|M N(t)|^{2} d t
$$




$$
P=\int_{T_{0}}^{T}|M N(t)|^{2} \frac{d t}{|t+i|^{2}} \ll M^{2} N^{2} T_{0}^{-1}
$$

(trivially) and, by the classical mean-value theorem (2.6),

$$
Q=\int_{T_{0}}^{T}|M N(t)|^{2} d t \ll\left(1+\frac{T}{M N}\right) M^{2} N^{2} \log ^{3} X
$$

(see also Lemma 2.1). We now have

$$
\begin{gathered}
P L^{2} \ll X^{2} T_{0}^{-1} \leq X^{2-\varepsilon}, \quad Q L^{2} \ll \frac{T}{N} X^{2} \log ^{3} X, \\
Q L^{2}(\log L)^{2} T^{-2} \ll \frac{1}{T} X^{2} \log ^{5} X \ll_{\varepsilon} X^{2-\varepsilon / 2}
\end{gathered}
$$

and

so that certainly

$$
Q \ll \frac{T}{L^{2} N} X^{2} \log ^{3} X \ll_{\varepsilon} X^{2-\varepsilon / 2},
$$

$$
H=Z L(\log T)^{2}+O_{\varepsilon}\left(X^{2-\varepsilon / 3}\right)
$$

We complete the proof of the proposition by showing that the first term on the right can be hidden in the $O_{\varepsilon}$-notation of the second term.

By the Cauchy-Schwarz inequality and a change of variable,

$$
Z^{2} \leq \int_{T_{0}+\tau}^{T+\tau}\left|\zeta\left(\frac{1}{2}+i t\right)\right|^{4}\left|N_{\tau}(t)\right|^{2} d t \int_{T_{0}}^{T}\left|M^{2} N(t)\right|^{2} d t
$$

where $\tau$ is some number from the interval $[-T, T]$ and $N_{\tau}(t)=N(t-\tau)$. With Lemma 2.1 at our disposal, Theorem 2 and the classical mean-value theorem (2.6) now yield

$$
\begin{aligned}
Z^{2} & \ll_{\varepsilon} T^{1+\varepsilon / 8} N\left(1+\frac{T}{M^{2} N}\right) M^{4} N^{2} \log ^{8} X \\
& \ll_{\varepsilon} X^{\varepsilon / 4}\left(\frac{T}{L^{2} N}+\frac{T^{2}}{X^{2}}\right) M^{4} N^{4} L^{2} \\
& \leq X^{\varepsilon / 4}\left(X^{-\varepsilon}+X^{-2 \varepsilon}\right) X^{4} L^{-2} \ll X^{4-3 \varepsilon / 4} L^{-2}
\end{aligned}
$$

and, as required, $Z L(\log T)^{2} \ll_{\varepsilon} X^{2-3 \varepsilon / 9}$.

Note that the conditions $N^{4} \leq T \leq X^{-\varepsilon} L^{2} N$ may be replaced with a single condition $L \geq X^{\varepsilon} T^{1 / 2}$. This follows from the case $N=1$ of the proposition, which was already known as a corollary of the fourth power moment for $\zeta(s)$ (see Harman [8], Lemma 6 and Titchmarsh [33], (7.6.2)).

3. Sums of the form $\sum_{m} a_{m} S\left(\mathcal{A}_{m}, X^{\phi}\right)$. By the Buchstab identity (1.6) we have two types of sum to consider. For the first type (the single 
sum) we need the following results from sieve methods.

LEMma 3.1 (Rosser-Iwaniec linear sieve). Let $\mathcal{B}$ be any finite integer sequence. Let $N \geq 0$ and for $d \geq 1$ define

$$
R(\mathcal{B}, d)=\# \mathcal{B}_{d}-N / d,
$$

where $\mathcal{B}_{d}=\{b \in \mathcal{B}: b \equiv 0(\bmod d)\}$. Let $D \geq z \geq 2, s=(\log D) / \log z$,

$$
P(z)=\prod_{p<z} p, \quad V=V(z)=\prod_{p<z}\left(1-\frac{1}{p}\right)
$$

and put $S=S(\mathcal{B}, z)=\#\{b \in \mathcal{B}:(b, P(z))=1\}$. Then, for $s \geq 1$,

$$
N V\left(1+O\left(e^{-s}\right)\right)+E_{-} \leq S \leq N V\left(1+O\left(e^{-s}\right)\right)+E_{+},
$$

where

$$
E_{ \pm}=\sum_{d<D} \mu_{d}(z) \varrho_{d}^{ \pm}(D) R(\mathcal{B}, d),
$$

with $\left(\varrho_{d}^{+}\right)$and $\left(\varrho_{d}^{-}\right)$being some sequences of 0 's and 1 's entirely determined by $D$, and with $\mu_{d}(z)=\mu(d)$, the Möbius function, if $d \mid P(z)$, but $\mu_{d}(z)=0$ otherwise.

Proof. This is an abridged version of a special case of [15], Theorem 1. In the statement of this theorem $E_{ \pm}$is given as a sum of absolute values, but it is clear from [15], Section 3 that the form of $E_{ \pm}$given here is valid. Mertens' Theorem

$$
V(z)=\frac{e^{-\gamma}}{\log z}+O\left((\log z)^{-2}\right)
$$

(see [6]) implies the inequality

$$
\prod_{w \leq p<z}\left(1-\frac{1}{p}\right)^{-1} \leq\left(\frac{\log z}{\log w}\right)\left(1+O\left(\frac{1}{\log w}\right)\right),
$$

which is the hypothesis [15], (1.3) with $\omega(p)=1$ (for all $p$ ) and $\kappa=1$. We remark, for future reference, that this inequality implies another:

$$
\sum_{w \leq p<z} \frac{1}{p} \leq \log \left(\frac{\log z}{\log w}\right)+O\left(\frac{1}{\log w}\right) .
$$

Lemma 3.2. Let $X^{\delta} \geq 2$ and suppose that $\left(a_{m}\right)$ is a finite $X^{\delta}$-sifted sequence with $a_{m} \geq 0$ for all $m$. Then, for $s \geq 1$,

$$
y \lambda\left(1+O\left(e^{-s}\right)\right)+R_{-} \leq \sum_{m} a_{m} S\left(\mathcal{A}_{m}, X^{\delta}\right) \leq y \lambda\left(1+O\left(e^{-s}\right)\right)+R_{+},
$$

where

$$
\lambda=V\left(X^{\delta}\right) \sum_{m} \frac{a_{m}}{m}
$$




$$
R_{ \pm}=\sum_{d<X^{s \delta}} \mu_{d}\left(X^{\delta}\right) \varrho_{d}^{ \pm}\left(X^{s \delta}\right) \sum_{m} a_{m} R(\mathcal{A}, d m)
$$

and

$$
R(\mathcal{A}, n)=\# \mathcal{A}_{n}-\frac{y}{n}=\left[\frac{x}{n}\right]-\left[\frac{x-y}{n}\right]-\frac{y}{n} .
$$

Proof. Lemma 3.1 is applied to the term $a_{m} S\left(\mathcal{A}_{m}, X^{\delta}\right)$ with $N=y / m$, and the result is summed over $m$. Note that when $a_{m} \mu_{d}\left(X^{\delta}\right) \neq 0$ we have $(m, d)=1$ and $R\left(\mathcal{A}_{m}, d\right)=R(\mathcal{A}, m d)$.

We remark that [16] permits a flexibility in arranging the above summation over $d$, which is often very useful, and was exploited by Harman in [8]. In our use of Lemma 3.2 however, we adopt Harman's later approach (see [9]) and ensure that the summation over $d$ is too short to play a significant rôle.

The next lemma gives a formula similar to the case $\phi=\delta$ of (1.5), but with the sequence $\left(a_{m}\right)$ having been replaced by a multiplicative convolution of two sequences $\left(a_{m}\right)$ and $\left(b_{n}\right)$.

Lemma 3.3. Let $0<8 \Delta<\theta<1 / 2$ and put $\delta=\Delta^{2}$. Let $X^{\delta} \geq 2$ and suppose that $\left(a_{m}\right)$ and $\left(b_{n}\right)$ are $X^{\delta}$-sifted sequences with $a_{m}=0$ for $m>M, b_{n}=0$ for $n>N$ and $0 \leq a_{m}, b_{n} \leq 1$ for all $m$ and $n$. Suppose further that

$$
N^{4} \leq X^{1-\theta+\Delta} \quad \text { and } \quad M^{2} N \leq X^{1+\theta-4 \Delta} .
$$

Then, for $X^{\theta} \leq Y \leq X / 2$,

$$
\sum_{m} a_{m} \sum_{n} b_{n} S\left(\mathcal{A}_{m n}, X^{\delta}\right)=y \lambda\left(1+O\left(e^{-1 / \Delta}\right)\right)+\bar{O}_{\Delta}\left(Y X^{-\Delta / 7}\right)
$$

with

$$
\lambda=V\left(X^{\delta}\right) \sum_{m} \sum_{n} \frac{a_{m} b_{n}}{m n} .
$$

Pr o of. By Lemma 3.2 with $s=1 / \Delta$ the proof reduces to the estimation of two sums, $R_{+}$and $R_{-}$. These can each be split into $O\left(\log ^{3} X\right)$ subsums of the form:

$$
\begin{array}{r}
R_{ \pm}\left(D, M_{1}, N_{1}\right) \\
=\sum_{D / 2<d<D} \mu_{d}\left(X^{\delta}\right) \varrho_{d}^{ \pm}\left(X^{\Delta}\right) \sum_{M_{1} / 2<m \leq M_{1}} \sum_{N_{1} / 2<n \leq N_{1}} a_{m} b_{n} R(\mathcal{A}, d m n),
\end{array}
$$

with $D \leq X^{\Delta}, M_{1} \leq M$ and $N_{1} \leq N$. Using (1.4) one can show that the lemma is a consequence of the bound

$$
R_{ \pm}\left(D, M_{1}, N_{1}\right)=\bar{O}_{\Delta}\left(Y X^{-\Delta / 6}\right) .
$$


To prove (3.3) we begin by writing $R_{ \pm}\left(D, M_{1}, N_{1}\right)$ as a sum over $k=$ $d m n$, so that it assumes the same form as the sum in Lemma 2.3. The relevant $\eta_{k}$ 's will all be $O_{\delta}(1)$, since $\mu_{d}\left(X^{\delta}\right)=0$ unless $d$ is a product of primes all less than $X^{\delta}$ while $\left(a_{m}\right)$ and $\left(b_{n}\right)$ are both $X^{\delta}$-sifted sequences (see (2.9)). On taking $K=D M_{1} N_{1}, K_{1}=K / 8, T_{0}=X^{\Delta}$ and $T=X^{1-\theta+\Delta}$, Lemma 2.3 yields

$$
\frac{1}{y}\left|R_{ \pm}\left(D, M_{1}, N_{1}\right)\right| \leq|f(x, y)|+|f(x, z)|+O_{\delta}\left(\left(X^{-\Delta}+X^{\Delta} L^{-1 / 2}\right) \log ^{2} X\right),
$$

where $L=X / K$ and $f(x, y)$ is as in Lemma 2.2 with

$$
\begin{aligned}
H(t)= & \sum_{D / 2<d \leq D} \mu_{d}\left(X^{\delta}\right) \varrho_{d}^{ \pm}\left(X^{\Delta}\right) \sum_{M_{1} / 2<m \leq M_{1}} a_{m} \\
& \times \sum_{N_{1} / 2<n \leq N_{1}} b_{n} \sum_{L / 4<l \leq 8 L}(d m n l)^{i t} \\
= & D(t) M_{1}(t) N_{1}(t) L(t) \quad \text { (say). }
\end{aligned}
$$

We have $N_{1}^{4} \leq N^{4} \leq X^{1-\theta+\Delta}=T$ and

$$
X^{-\Delta} L^{2} N_{1}=X^{2-\Delta} / D^{2} M_{1}^{2} N_{1} \geq X^{2-3 \Delta} / M^{2} N \geq T .
$$

Hence Proposition 2.2 applies to give

$$
\int_{T_{0}}^{T}|H(t)|^{2} d t \ll \Delta X^{2-\Delta / 3} .
$$

This shows, by Lemma 2.4, that $f(x, y)$ and $f(x, z)$ are both $\bar{O}_{\Delta}\left(X^{-\Delta / 6}\right)$. The bound (3.3) and the lemma both follow as $L \geq T^{3 / 8} \geq X^{3 / 16}>X^{3 \Delta}$.

Lemma 3.3 can give useful formulae for the single sum on the right-hand side of (1.6). Lemma 3.4 (below) treats the double sum. These two results are combined in Proposition 3.1, which is central to much of what follows.

Lemma 3.4. Let $0<\delta<1 /(2 k+2) \leq \theta<1 /(2 k)$ and set $\phi=\theta+$ $(1-\theta) /(2 k-1)-\delta$ with $k$ some integer satisfying $k \geq 4$. Let $X^{\delta} \geq 4$, $A \geq 3$ and suppose that $\left(a_{m}\right)$ is an $X^{\phi}$-sifted sequence with $0 \leq a_{m} \leq 1$, for all $m$, and $a_{m}=0$, for $m>X^{1-2 \phi-\delta}$. Then, for $X^{\theta}(\log X)^{3 A+1} \leq Y \leq$ $X(\log X)^{1-3 A}$, we have

$$
\begin{aligned}
\sum_{m} a_{m} \sum_{X^{\delta} \leq p<X^{\phi}} S\left(\mathcal{A}_{m p}, p\right) & \\
& =y \lambda(x)+O_{\delta, A}\left(\frac{Y}{\log ^{2} X} \sum_{m} \frac{a_{m}}{m}\right)+\bar{O}_{\delta, A}\left(Y(\log X)^{3-A}\right),
\end{aligned}
$$

where $\lambda(x)$ does not depend on $Y$. 
Pr o of. We begin by rewriting the sum over $m$ and $p$ as

$$
\Sigma=\sum_{m} a_{m} \sum_{X^{\delta} \leq p<X^{\phi}} \sum_{l} S(\{l\}, p) \xi(m p l),
$$

where $S(\{l\}, p)$ is as in Lemma 3.1 and $\xi(u)$ is the characteristic function of the interval $(x-y, x]$. An equivalent form of (1.6) is the identity

$$
S(\{l\}, z)=S(\{l\}, w)-\sum_{\substack{q \mid l \\ w \leq q<z}} S(\{l / q\}, q),
$$

for $0 \leq w \leq z$. Hence,

$$
\begin{aligned}
\Sigma= & \sum_{m} a_{m} \sum_{X^{\delta} \leq p<X^{\phi}} \sum_{l} S\left(\{l\}, d_{p}^{-}\right) \xi(m p l) \\
& -\sum_{m} a_{m} \sum_{X^{\delta} \leq p<X^{\phi}} \sum_{d_{p}^{-} \leq q<p} \sum_{r} S(\{r\}, q) \xi(m p q r) \\
= & \Sigma^{-}-\sigma^{-} \quad \text { (say), }
\end{aligned}
$$

where $d_{n}^{-}=2^{b}$ with $b$ the integer such that $2^{b}<n \leq 2^{b+1}$. Similarly, with $d_{n}^{+}=2 d_{n}^{-}$,

$$
\begin{aligned}
\Sigma^{+} & =\sum_{m} a_{m} \sum_{X^{\delta} \leq p<X^{\phi}} \sum_{l} S\left(\{l\}, d_{p}^{+}\right) \xi(m p l) \\
& =\Sigma-\sum_{m} a_{m} \sum_{X^{\delta} \leq p<X^{\phi}} \sum_{p \leq q<d_{p}^{+}} \sum_{r} S(\{r\}, q) \xi(m p q r) \\
& =\Sigma-\sigma^{+} \quad \text { (say). }
\end{aligned}
$$

The sums $\Sigma^{ \pm}$above are subdivided into $O\left(\log ^{2} X\right)$ sums of the form

$$
\Sigma\left(M_{1}, P\right)=\sum_{M_{1} / 2<m \leq M_{1}} a_{m} \sum_{\substack{P / 2<p \leq P \\ X^{\delta} \leq p<X^{\phi}}} \sum_{l} S(\{l\}, Q) \xi(m p l),
$$

where $M_{1}$ and $P$ are integer powers of 2 and $Q=d_{P}^{ \pm}$. What has been achieved is a separation of the variable $p$ by means of butchery, rather than through a more precise application of Perron's formula (as in [8], formula $(25))$.

To each sum $\Sigma\left(M_{1}, P\right)$ we now apply Lemma 2.2 with

$$
T_{0}=(\log X)^{A}, \quad T=X^{1-\theta}(\log X)^{-2 A-1} .
$$

For $Y$ in the assumed range, this gives

$$
\frac{1}{y} \Sigma\left(M_{1}, P\right)=\lambda(x)+f(x, y)+O\left((\log X)^{1-A}\right),
$$


where $\lambda(x)$ does not depend on $Y$. Here $f(x, y)$ is given in terms of the Dirichlet polynomial

$$
\begin{aligned}
H(t) & =\sum_{M_{1} / 2<m \leq M} a_{m} \sum_{\substack{P / 2<p \leq P \\
X^{\delta} \leq p<X^{\phi}}} \sum_{L / 4<l \leq 4 L} S(\{l\}, Q)(m p l)^{i t} \\
& =M_{1}(t) P(t) L(t) \quad(\text { say }),
\end{aligned}
$$

with $L=X / M_{1} P$. Note that here $Q \geq X^{\delta} / 2 \geq X^{\delta / 2}$, so that, by (2.9), the coefficients $\eta_{h}$ of $H(t)$ are all $O_{\delta}(1)$.

We now appeal to Proposition 2.1 with $M(t)=P(t)$ and $N(t)=$ $M_{1}(t) L(t)$. The bound (2.10) is supplied by (1.7) with $B=1 / \delta$. The relevant sequences are $X^{\delta / 2}$-sifted and bounded by $O_{\delta}(1)$. Furthermore we have $P<2 X^{\phi}$. Hence, assuming that $X$ is sufficiently large in terms of $\delta$ and $A$, Proposition 2.1 yields

$$
\int_{T_{0}}^{T}|H(t)|^{2} d t \ll_{\delta, A} X^{2}(\log X)^{1-2 A} .
$$

This shows that $f(x, y)=\bar{O}_{\delta, A}\left((\log X)^{1-A}\right)$ (see (2.5) and Lemma 2.4).

Collecting our results for all the $\Sigma\left(M_{1}, P\right)$ 's we find (using (1.4))

$$
\Sigma=\Sigma^{ \pm} \pm \sigma^{ \pm}=y \lambda^{ \pm}(x) \pm \sigma^{ \pm}+\bar{O}_{\delta, A}\left(Y(\log X)^{3-A}\right),
$$

with $\lambda^{+}(x)$ and $\lambda^{-}(x)$ independent of $Y$. Hence,

$$
\lambda^{-}(x)-\lambda^{+}(x)=\frac{\sigma^{+}+\sigma^{-}}{y}+\bar{O}_{\delta, A}\left((\log X)^{3-A}\right) .
$$

For all $Y$ we have,

$$
0 \leq \sigma^{+}+\sigma^{-} \leq \sum_{m} a_{m} \sum_{X^{\delta} \leq p<X^{\phi}} \sum_{p / 2 \leq q<2 p} S\left(\mathcal{A}_{m p q}, X^{\delta / 2}\right) .
$$

To the right-hand side we apply Lemma 3.2, setting $s=1$ and bounding the remainder terms $R_{ \pm}$trivially by $|R(\mathcal{A}, n)|<1$. This yields the upper bound

$$
\begin{aligned}
& \sigma^{+}+\sigma^{-} \ll y V\left(X^{\delta / 2}\right) \sum_{m} \sum_{X^{\delta} \leq p<X^{\phi}} \sum_{p / 2 \leq q<2 p} \frac{a_{m}}{m p q} \\
& +X^{\delta / 2} \sum_{m} \sum_{X^{\delta} \leq p<X^{\phi}} \sum_{p / 2 \leq q<2 p} a_{m} \\
& \ll_{\delta}\left(\frac{Y}{\log ^{2} X}+\frac{X^{1-\delta / 2}}{\log X}\right) \sum_{m} \frac{a_{m}}{m},
\end{aligned}
$$

by the implicit bound $m p q<2 X^{1-\delta}$ (see the hypotheses of the lemma) followed by applications of (3.1) and (3.2). Therefore, when $Y=X(\log X)^{1-3 A}$ 
(say) we will have

$$
\frac{\sigma^{+}+\sigma^{-}}{y} \ll_{\delta, A} \frac{1}{\log ^{2} X} \sum_{m} \frac{a_{m}}{m} .
$$

Returning with this to (3.6) one finds that, for all $Y$ in the assumed range,

$$
\sigma^{+}+\sigma^{-}=O_{\delta, A}\left(\frac{Y}{\log ^{2} X} \sum_{m} \frac{a_{m}}{m}\right)+\bar{O}_{\delta, A}\left(Y(\log X)^{3-A}\right) .
$$

Since $\sigma^{+}$and $\sigma^{-}$are both non-negative, one can replace $\sigma^{+}+\sigma^{-}$with $\sigma^{ \pm}$ here. Then (3.5), with either choice of sign, yields the stated result of the lemma.

Proposition 3.1. Let $0<8 \Delta<1 /(2 k+2) \leq \theta<1 /(2 k), \delta=\Delta^{2}$ and $\phi=\theta+(1-\theta) /(2 k-1)-\delta$ with $k$ some integer satisfying $k \geq 6$. Let $X^{\delta} \geq 4, A \geq 3$ and suppose that $\left(a_{m}\right)$ and $\left(b_{n}\right)$ are $X^{\phi}$-sifted sequences with $a_{m}=0$ for $m>M, b_{n}=0$ for $n>N$, and $0 \leq a_{m}, b_{n} \leq 1$ for all $m$ and $n$. Then, provided that

$$
N^{4} \leq X^{1-\theta+\Delta} \quad \text { and } \quad M^{2} N \leq X^{1+\theta-4 \Delta},
$$

we have

$$
\begin{aligned}
\sum_{m} a_{m} \sum_{n} & b_{n} S\left(\mathcal{A}_{m n}, X^{\phi}\right) \\
= & y \lambda(x)+O\left(\frac{\delta Y}{\log X} \sum_{m} \sum_{n} \frac{a_{m} b_{n}}{m n}\right)+\bar{O}_{\delta, A}\left(Y(\log X)^{3-A}\right),
\end{aligned}
$$

for $X^{\theta}(\log X)^{3 A+1} \leq Y \leq X(\log X)^{1-3 A}$, with $\lambda(x)$ independent of $Y$.

Proof. First observe that the sequence $\left(a_{m}^{*}\right)$ given by

$$
a_{m}^{*}=\sum_{r n=m} a_{r} b_{n}
$$

is $X^{\phi}$-sifted, satisfies $a_{m}^{*}=0$ for $m>M N$ and $0 \leq a_{m}^{*} \ll_{\delta} 1$ otherwise. In particular we can replace all the $a_{m}$ 's in Buchstab's identity (1.6) with $a_{m}^{*}$ 's. Then, to the first sum on the right-hand side, we can immediately apply Lemma 3.3. The remaining double sum can be dealt with by Lemma 3.4 , since we have $M N \leq X^{1-\Delta-3(1-\theta) / 8}$ where $\Delta>\delta$ and $3(1-\theta) / 8>2 \phi$ (by virtue of the condition $k \geq 6$ ). Hence

$$
\begin{aligned}
\sum_{m} a_{m}^{*} S\left(\mathcal{A}_{m}, X^{\phi}\right)= & y\left(\lambda-\lambda_{1}(x)\right)+O\left(Y \lambda e^{-1 / \Delta}\right) \\
& +O_{\delta, A}\left(\frac{Y}{\log ^{2} X} \sum_{m} \frac{a_{m}^{*}}{m}\right) \\
& +\bar{O}_{\Delta}\left(Y X^{-\Delta / 7}\right)+\bar{O}_{\delta, A}\left(Y(\log X)^{3-A}\right)
\end{aligned}
$$


in the stated range for $Y$. Here

$$
\lambda=V\left(X^{\delta}\right) \sum_{m} \frac{a_{m}^{*}}{m} \ll(\delta \log X)^{-1} \sum_{m} \frac{a_{m}^{*}}{m},
$$

by (3.1). The proposition follows since $\delta^{-1} e^{-1 / \Delta}=\Delta^{-2} e^{-1 / \Delta} \ll \Delta^{2}=\delta$, $X^{\Delta / 7} \gg_{\Delta, A} \log ^{A} X$, and $O_{\delta, A}(1 / \log X) \ll \delta+O_{\delta, A}\left((\log X)^{-A}\right)$.

4. A negative constant. In this section we consider the sums $\Sigma_{1}$ and $\Sigma_{2}$ from (1.8). We assume henceforth that $\theta, \phi, \Delta, \delta$ and $X$ are as in Proposition 3.1 and that $E \geq 1$. By (3.2), Proposition 3.1 applies to give

$$
\Sigma_{i}=y \lambda_{i}(x)+O_{\theta}\left(\frac{\delta Y}{\log X}\right)+\bar{O}_{\delta, E}\left(Y(\log X)^{-E}\right)
$$

for

$$
X^{\theta}(\log X)^{3 E+10} \leq Y \leq X(\log X)^{-3 E-8}
$$

and $i=1,2$. We shall show independently that when $A \geq 1$ and $Y=$ $X(\log X)^{-A}$ one has

$$
\Sigma_{i}=c_{i} \frac{y}{\log X}+O_{A, \delta}\left(Y(\log X)^{-2}\right)
$$

for $X / 2<x<X$ and $i=1,2$, with constants $c_{1}, c_{2}$ depending only on $\phi$.

Lemma 4.1 (Buchstab-Selberg). Let $A \geq 1, y>0, x-y \geq z \geq 2$ and $s=(\log x) / \log z \leq n$, where $n$ is an integer. Take $\mathcal{A}=(x-y, x]$. Then, for $y \leq x(\log x)^{-A}$,

$$
S(\mathcal{A}, z)=\omega(s) \frac{y}{\log z}+O_{A, n}\left(\frac{x}{(\log x)^{2 A+2}}\right),
$$

where $\omega(s)$ is the continuous real function on $[1, \infty)$ satisfying $s \omega(s)=1$, for $1 \leq s \leq 2$, and $(s \omega(s))^{\prime}=\omega(s-1)$, for $s>2$.

Proof. This can be proved by induction on $n$ (as in [2]). The case $n=2$ is just the prime number theorem (1.9). For $n \geq 3$ the induction step can be accomplished by means of a Buchstab identity such as (1.6).

LEMmA 4.2. Let $\varepsilon>0,2 \leq a \leq b / 2, a \geq b^{\varepsilon}$, and suppose that $f(x)$ is $a$ positive valued, piecewise continuously differentiable function on $[a, b]$ with

$$
\frac{d}{d x} \log f(x)=O\left(\frac{1}{x}\right)
$$

except at the finitely many points where $f^{\prime}(x)$ is undefined. Then, for any $A \geq 1$,

$$
\sum_{a \leq p<b} f(p)=\left(1+O_{A, \varepsilon}\left((\log a)^{-A}\right)\right) \int_{a}^{b} \frac{f(x)}{\log x} d x .
$$


Proof. This is a standard result and follows quite easily from the prime number theorem (1.9) through integration by parts. gives

When $Y=X(\log X)^{-A}$, with $A \geq 1$, and $X / 2<x<X$, Lemma 4.1

$$
\Sigma_{1}=S\left(\mathcal{A}, X^{\phi}\right)=\omega\left(\frac{\log x}{\phi \log X}\right) \frac{y}{\phi \log X}+O_{A, \delta}\left(\frac{x}{(\log x)^{2 A+2}}\right) .
$$

As $\delta<\phi<1 / 6$ and $(\log x) / \log X=1+O(1 / \log X)$, and as $\omega(s)$ is continuous for $s \geq 1$, this yields the case $i=1$ of (4.3) with $c_{1}=\omega(1 / \phi) / \phi$. Similarly,

$$
\begin{aligned}
\Sigma_{2} & =\sum_{X^{\phi} \leq p<X^{1 / 2}} S\left(\mathcal{A}_{p}, X^{\phi}\right) \\
& =\sum_{X^{\phi} \leq p<X^{1 / 2}} \omega(s) \frac{(y / p)}{\phi \log X}\left(1+O_{A, \delta}\left(\frac{1}{\log X}\right)\right),
\end{aligned}
$$

where $s=s(p)=(1-(\log p) /(\log X)) / \phi$. Here we apply Lemma 4.2 to obtain

$$
\sum_{X^{\phi} \leq p<X^{1 / 2}} \frac{\omega(s)}{\phi p}=\left(1+O_{\delta}\left(\frac{1}{\log X}\right)\right) \int_{X^{\phi}}^{X^{1 / 2}} \frac{\omega(s(u))}{\phi u \log u} d u .
$$

A change of variables now yields the case $i=2$ of (4.3) with

$$
c_{2}=\int_{1 /(2 \phi)}^{1 / \phi-1} \frac{\omega(s)}{1-\phi s} d s .
$$

By comparing (4.1) and (4.3) at $Y=X(\log X)^{-3 E-8}$, we find that

$$
\lambda_{i}(x)=\frac{c_{i}}{\log X}+O_{\theta}\left(\frac{\delta}{\log X}\right)+\bar{O}_{\delta, E}\left((\log X)^{-E}\right) .
$$

Substituting (4.1) and (4.4) into (1.8) one obtains

$$
S-R=\left(1-c_{3}+O_{\theta}(\delta)\right) \frac{y}{\log X}+\bar{O}_{\delta, E}\left(Y(\log X)^{-E}\right),
$$

for $Y$ satisfying (4.2) and $c_{3}=1-\left(c_{1}-c_{2}\right)$. Integrating by parts and making use of the definition of $\omega(s)$, given in Lemma 4.1, we have

$$
\begin{aligned}
c_{3} & =2 \omega(2)-\frac{1}{\phi} \omega\left(\frac{1}{\phi}\right)+\int_{1 /(2 \phi)}^{1 / \phi-1} \frac{\omega(s)}{1-\phi s} d s \\
& =\int_{1}^{1 /(2 \phi)-1} \omega(s) \log (s+1) d s+\int_{1 /(2 \phi)-1}^{1 / \phi-2} \omega(s) \log (1 / \phi-1-s) d s
\end{aligned}
$$




$$
=\int_{\alpha=\phi}^{1 / 2} \int_{\beta=\phi}^{\alpha} \omega\left(\frac{1-\alpha-\beta}{\beta}\right) \frac{d \alpha d \beta}{\alpha \beta^{2}},
$$

where $\omega(s)=0$ for $s<1$ (so that a condition $\alpha+2 \beta \leq 1$ is implied). For an alternative proof of this apply Lemmas 4.1 and 4.2 to $R$ and $S$ in (1.8).

To prepare for the numerical estimation of $c_{3}$, or one of the many other constants that we shall define in terms of integrals involving $\omega(s)$, one can use either $(s \omega(s))^{\prime}=\omega(s-1)$ (with integration by parts) or explicit formulae for $\omega(s)$, got by iterating

$$
s \omega(s)=1+\int_{1}^{s-1} t \omega(t) \frac{d t}{t} \quad(s>2) .
$$

For $\theta=1 / 14$ the largest value of $s$ encountered will be $(1-2 \phi) / \phi=53 / 12$ $<5$. Hence it is practical to write down all the constants in terms of multiple or (more often) single integrals that can be estimated by machine calculation. We shall be content to give only the results of these calculations (to four decimal places, unrounded), leaving out all details of the intervening preparation and calculation.

Suppose now that $\theta=1 / 14$. Then for Proposition 3.1 we must have $k=6$ and $\phi=12 / 77-\delta$. Unfortunately, with $\delta=0$,

$$
c_{3}=1.5965 \ldots \text {, }
$$

so that $c_{1}-c_{2}=1-c_{3}$ is negative and (4.5) in conjuction with the trivial bound $R \geq 0$ gives nothing. For a comparison, when $\theta=1 / 12$ and $\phi=5 / 27$ the value of $c_{3}$ is approximately 0.9825 , and when $\theta=1 / 16$ and $\phi=7 / 52$ it is about 2.302 .

In the next section we apply Proposition 3.1 to special subsums of the sum $R$ in (1.8). This recovers certain regions of the integral over $\alpha$ and $\beta$ in (4.6). Some more regions are recovered by virtue of Lemma 2.7. The proof that we recover enough to get Theorem 1 is given in Section 6 .

5. Sums of the form $\sum_{p} \sum_{q} a_{p q} S\left(\mathcal{A}_{p q}, q\right)$. We give five (really three) lemmas for dealing with such sums. Lemmas 5.1 and 5.3 depend on Proposition 3.1. Their applications in the case $\theta=1 / 14$ are worked out in Lemmas 5.2 and 5.4 (respectively). The section ends with some applications of Lemma 5.5, which is a corollary of the simple Lemma 2.7. We continue to assume that $\theta, \phi, \Delta, \delta$ and $X$ are as in Proposition 3.1 and that $E \geq 1$.

Lemma 5.1. Let

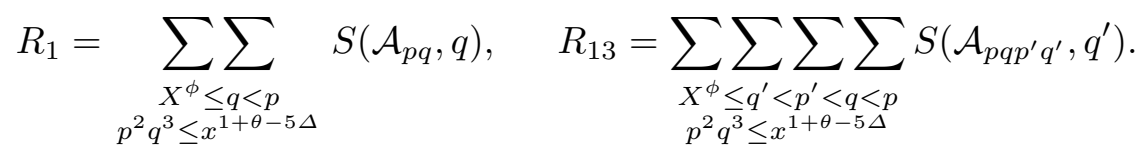


Then, for $X^{\theta}(\log X)^{3 E+13} \leq Y \leq X(\log X)^{-3 E-11}$, we have

$$
R_{1}-R_{13}=y \lambda(x)+O_{\theta}\left(\frac{\delta Y}{\log X}\right)+\bar{O}_{\delta, E}\left(Y(\log X)^{-E}\right),
$$

where $\lambda(x)$ is independent of $Y$.

Proof. By two applications of the Buchstab identity (1.6),

$$
R_{1}=R_{11}-R_{12}+R_{13}
$$

where

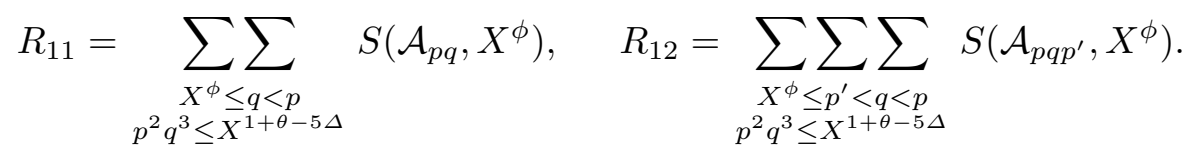

For $j=1,2$, we have

$$
R_{1 j}^{+}=\sum_{Q} R_{1 j}(Q, 1) \leq R_{1 j} \leq \sum_{Q} R_{1 j}(Q, 2)=R_{1 j}^{-},
$$

where $Q$ runs over the positive integer powers of 2 ,

$$
\begin{gathered}
R_{11}(Q, C)=\sum_{Q / 2<q \leq Q} \sum_{Q / C<p \leq f(Q / C)} S\left(\mathcal{A}_{p q}, X^{\phi}\right), \\
R_{12}(Q, C)=\sum_{p^{\prime} \leq C Q / 2} \sum_{Q / 2<q \leq Q} \sum_{Q / C<p \leq f(Q / C)} S\left(\mathcal{A}_{p q p^{\prime}}, X^{\phi}\right),
\end{gathered}
$$

and

$$
f(u)=X^{(1+\theta-5 \Delta) / 2} u^{-3 / 2} .
$$

Taking account of the implicit conditions, $p, q, p^{\prime} \geq X^{\phi}$, at most $O(\log X)$ of the sums $R_{1 j}(Q, C)$ in (5.2) are non-empty, and which ones those are does not depend on $Y$. To such sums we apply Proposition 3.1 with $A=E+4$. Since $Y$ is in the right range, and since the equation $p p^{\prime}=m$ has at most two solutions (for any given $m$ ), we just have to check that

$$
Q^{4} \leq X^{1-\theta+\Delta} \quad \text { and } \quad(f(Q / C) C Q / 2)^{2} Q \leq X^{1+\theta-4 \Delta} .
$$

In fact for non-empty sums, $Q / C<f(Q / C)=X^{(1+\theta-5 \Delta) / 2}(Q / C)^{-3 / 2}$ and

$$
Q<C X^{(1+\theta-5 \Delta) / 5} \leq X^{(1+\theta) / 5} .
$$

As $(1-\theta) / 4-(1+\theta) / 5=(1-9 \theta) / 20>1 / 80$ this easily gives the first of the above conditions. For the second, $(f(Q / C) C Q / 2)^{2} Q=X^{1+\theta-5 \Delta} C^{5} / 4$ and $C^{5} / 4 \leq 8 \leq X^{\Delta}$.

By (3.2) with $z=Q, w=Q / 2$ or $z=X, w=X^{\phi}$, one writes down the result of Proposition 3.1 as

$$
R_{1 j}(Q, C)=y \lambda_{j}(Q, C ; x)+O_{\theta}\left(\delta Y(\log X)^{-2}\right)+\bar{O}_{\delta, E}\left(Y(\log X)^{-E-1}\right),
$$


with $\lambda_{j}(Q, C ; x)$ independent of $Y$. Hence (see (1.4)) the uppermost and lower bounds in (5.2) satisfy

$$
R_{1 j}^{ \pm}=y \lambda_{j}^{ \pm}(x)+O_{\theta}\left(\frac{\delta Y}{\log X}\right)+\bar{O}_{\delta, E}\left(Y(\log X)^{-E}\right),
$$

with $\lambda_{j}^{ \pm}(x)$ independent of $Y$. From this we conclude that, for $j=1,2$ and $Y$ in the assumed range,

$$
R_{1 j}^{-}-R_{1 j}^{+}=y\left(\lambda_{j}^{-}(x)-\lambda_{j}^{+}(x)\right)+O_{\theta}\left(\frac{\delta Y}{\log X}\right)+\bar{O}_{\delta, E}\left(Y(\log X)^{-E}\right) .
$$

It is now of interest to bound $\left|\lambda_{j}^{-}(x)-\lambda_{j}^{+}(x)\right|$ on average over $x$, so that we may control the $O$-terms in the equations

$$
R_{1 j}=R_{1 j}^{+}+O\left(R_{1 j}^{-}-R_{1 j}^{+}\right),
$$

from (5.2). Firstly observe that $R_{1 j}(Q, 2)-R_{1 j}(Q, 1)$ is a sum of terms, $S\left(\mathcal{A}_{p q}, X^{\phi}\right)$ or $S\left(\mathcal{A}_{p q p^{\prime}}, X^{\phi}\right)$, with $q \asymp Q$ and either $p \asymp Q, p \asymp f(Q)$, or $p^{\prime} \asymp$ $Q$. For an upper bound we can replace $X^{\phi}$ in each term by $z_{1}=(x /(p q))^{\phi}$ or $z_{2}=\left(x /\left(p q p^{\prime}\right)\right)^{\phi}$, as appropriate. Then, at $Y=X(\log X)^{-3 E-11}$, Lemma 4.1 applies to give

$$
\begin{aligned}
S\left(\mathcal{A}_{p q}, z_{1}\right) & =\frac{1}{\phi} \omega\left(\frac{1}{\phi}\right) \frac{y /(p q)}{\log (x /(p q))}+O_{\delta, E}\left(\frac{x /(p q)}{(\log (x /(p q)))^{6 E+24}}\right) \\
& \ll_{\delta, E} \frac{Y /(p q)}{\log X} ; \\
S\left(\mathcal{A}_{p q p^{\prime}}, z_{2}\right) & \ll_{\delta, E} \frac{Y /\left(p q p^{\prime}\right)}{\log X}
\end{aligned}
$$

(note that we may assume $p q p^{\prime} \ll p q^{2} \ll X^{1+\theta-2 \phi}$, so that $X /\left(p q p^{\prime}\right) \gg X^{\phi}$ ).

Hence, for this $Y$, and $j=1,2$,

$$
R_{1 j}(Q, 2)-R_{1 j}(Q, 1) \ll_{\delta, E} Y(\log X)^{-3}
$$

(see $(3.2))$, and

$$
R_{1 j}^{-}-R_{1 j}^{+}=O_{\delta, E}\left(Y(\log X)^{-2}\right) \ll\left(\delta+O_{\delta, E}\left((\log X)^{-E}\right)\right) \frac{Y}{\log X} .
$$

Returning to (5.4), one obtains the desired bound:

$$
\lambda_{j}^{-}(x)-\lambda_{j}^{+}(x)=O_{\theta}\left(\frac{\delta}{\log X}\right)+\bar{O}_{\delta, E}\left((\log X)^{-E}\right) .
$$

By (5.4) again,

$$
R_{1 j}^{-}-R_{1 j}^{+}=O_{\theta}\left(\frac{\delta Y}{\log X}\right)+\bar{O}_{\delta, E}\left(Y(\log X)^{-E}\right),
$$

for $j=1,2$ and all $Y$ satisfying the hypotheses of the lemma. The lemma now follows from (5.1), (5.5), (5.3) and the last equation. 
Lemma 5.2. Let $\theta=1 / 14$. Then, for $X^{1 / 14}(\log X)^{3 E+13} \leq Y \leq$ $X(\log X)^{-3 E-11}$, the sum $R_{1}$ in Lemma 5.1 satisfies

$$
R_{1} \geq\left(a_{I}-b_{I}+O(\delta)\right) \frac{y}{\log X}+\bar{O}_{\delta, E}\left(Y(\log X)^{-E}\right),
$$

where

$$
a_{I}=\int_{\beta=\phi}^{(1+\theta) / 5-\Delta} \int_{\alpha=\beta}^{(1+\theta-5 \Delta-3 \beta) / 2} \omega\left(\frac{1-\alpha-\beta}{\beta}\right) \frac{d \alpha d \beta}{\alpha \beta^{2}}
$$

and

$$
b_{I}=\iiint \int_{\substack{\phi<\sigma<\varrho<\beta<\alpha \\ 2 \alpha+3 \beta<1+\theta-5 \Delta}} \omega\left(\frac{1-\alpha-\beta-\varrho-\sigma}{\sigma}\right) \frac{d \alpha d \beta d \varrho d \sigma}{\alpha \beta \varrho \sigma^{2}}
$$

(with $\phi=12 / 77-\delta$ ).

Proof. We consider the sums

$$
R_{1}(P, Q)=\sum_{P / 2<p \leq P} \sum_{Q / 2<q \leq Q} S\left(\mathcal{A}_{p q}, q\right)
$$

with $P$ and $Q$ positive integer powers of 2 satisfying $Q \geq 2 X^{\phi}, P \geq 2 Q$ and $P^{2} Q^{3} \leq X^{1+\theta-5 \Delta}$. For $Y=X(\log X)^{-3 E-11}$, Lemmas 4.1 and 4.2 give

$$
\begin{aligned}
R_{1}(P, Q) & \sum_{P / 2<p \leq P} \sum_{Q / 2<q \leq Q}\left(\omega\left(\frac{\log (x /(p q))}{\log q}\right) \frac{y /(p q)}{\log q}\right. \\
& \left.+O_{E}\left(\frac{x /(p q)}{(\log (x /(p q)))^{6 E+24}}\right)\right) \\
= & \sum_{P / 2<p \leq P} \sum_{Q / 2<q \leq Q} \omega\left(\frac{\log (X /(P Q))}{\log Q}\right) \frac{y /(p q)}{\log Q}\left(1+O_{E}\left(\frac{1}{\log X}\right)\right) \\
= & \int_{u=P / 2} \int_{v=Q / 2}^{Q} \omega\left(\frac{\log (X /(P Q))}{\log Q}\right) \frac{(y /(u v)) d u d v}{(\log u)(\log v) \log Q}\left(1+O_{E}\left(\frac{1}{\log X}\right)\right) \\
= & \left(1+O_{E}\left(\frac{1}{\log X}\right)\right) y \int_{u=P / 2}^{P} \int_{v=Q / 2}^{Q} \omega\left(\frac{\log (X /(u v))}{\log v}\right) \frac{d u d v}{u v(\log u)(\log v)^{2}} .
\end{aligned}
$$

Summing over $P$ and $Q$, we obtain the lower bound, 


$$
\begin{aligned}
R_{1} \geq & \left(1+O_{E}\left(\frac{1}{\log X}\right)\right) \\
& \times \frac{y}{\log X} \quad \underset{\substack{X^{\phi} \leq v \leq u \\
u^{2} v^{3} \leq X^{1+\theta-5 \Delta}}}{\int} \omega\left(\frac{\log (X /(u v))}{\log v}\right) \frac{(\log X) d u d v}{u v(\log u)(\log v)^{2}}
\end{aligned}
$$

(that part of the integral, where either $v \asymp X^{\phi}, u \asymp v$ or $u^{2} v^{3} \asymp X^{1+\theta-5 \Delta}$, is accounted for by the $O$-term). By the change of variables, $u=X^{\alpha}$ and $v=X^{\beta}$, the integral is seen to equal $a_{I}$.

In the sum $R_{13}$ in Lemma 5.1, one has

$$
p q p^{\prime} q^{\prime}<x^{(4 / 5)(1+\theta)}=x^{6 / 7}<x-y,
$$

so that $\mathcal{A}$ contains no integer of the form $1 p q p^{\prime} q^{\prime}$, and we may actually assume

$$
x /\left(p q p^{\prime} q^{\prime}\right) \geq q^{\prime} \geq X^{\phi} .
$$

Hence, for $Y=X(\log X)^{-3 E-11}$, Lemma 4.1 gives

$$
\begin{aligned}
& S\left(\mathcal{A}_{p q p^{\prime} q^{\prime}}, q^{\prime}\right) \\
& \quad \leq S\left(\mathcal{A}_{p q p^{\prime} q^{\prime}}, q^{\prime} / 2\right) \\
& \quad=\omega\left(\frac{\log \left(x /\left(p q p^{\prime} q^{\prime}\right)\right)}{\log \left(q^{\prime} / 2\right)}\right) \frac{y /\left(p q p^{\prime} q^{\prime}\right)}{\log \left(q^{\prime} / 2\right)}+O_{E}\left(\frac{x /\left(p q p^{\prime} q^{\prime}\right)}{\left(\log \left(x /\left(p q p^{\prime} q^{\prime}\right)\right)\right)^{6 E+24}}\right) .
\end{aligned}
$$

By an argument similar to the one used above (for the lower bound on $R_{1}$ ) we deduce that

$$
R_{13} \leq\left(b_{I}+O_{E}\left(\frac{1}{\log X}\right)\right) \frac{y}{\log X}
$$

when $Y=X(\log X)^{-3 E-11}$. Note that the condition $\alpha+\beta+\varrho+2 \sigma \leq 1$, implicit in the definition of $b_{I}$, comes from (5.6).

We can now conclude that

$$
R_{1}-R_{13} \geq\left(a_{I}-b_{I}+O_{E}\left(\frac{1}{\log X}\right)\right) \frac{y}{\log X},
$$

for $X / 2<x<X$ and $Y=X(\log X)^{-3 E-11}$. Together with the result of Lemma 5.1, this gives

$$
a_{I}-b_{I} \leq(\log X) \lambda(x)+O(\delta)+\bar{O}_{\delta, E}\left((\log X)^{1-E}\right) .
$$

By Lemma 5.1 again, it follows that

$$
\frac{y}{\log X}\left(a_{I}-b_{I}\right) \leq R_{1}-R_{13}+O\left(\frac{\delta Y}{\log X}\right)+\bar{O}_{\delta, E}\left(Y(\log X)^{-E}\right),
$$

for $X^{1 / 14}(\log X)^{3 E+13} \leq Y \leq X(\log X)^{-3 E-11}$. As $R_{13} \geq 0$, this completes the proof of the lemma. 
Our calculations (with $\theta=1 / 14, \phi=12 / 77$ and $\Delta=0$ ) yield

$$
c_{I}=a_{I}-b_{I}=0.3606 \ldots-0.0039 \ldots=0.3567 \ldots
$$

LEMMA 5.3. Let

$$
\begin{aligned}
R_{2}^{*}= & \sum_{p} \sum_{\substack{X^{\phi} \leq q \leq X^{(1-\theta) / 4} \\
R_{23}^{*}=}} \sum_{\substack{\frac{1}{16} X^{1-\theta+5} \leq q r^{2} \leq X^{1+\theta-5} \leq \\
X^{\phi} \leq p^{\prime}<q \leq X^{(1-\theta) / 4}}} \sum_{\substack{r^{\prime} \geq p^{\prime} \\
\frac{1}{16} X^{1-\theta+5 \Delta} \leq q\left(p^{\prime} r^{\prime}\right)^{2} \leq X^{1+\theta-5 \Delta}}} S(\{r\}, q) \xi(p q r), \\
& \times \sum_{\substack{q^{\prime} \geq X^{\phi} \\
r^{\prime} q p^{\prime}\left(q^{\prime}\right)^{2}<X}} \sum_{l^{\prime}} S\left(\left\{l^{\prime}\right\}, q^{\prime}\right) \xi\left(q p^{\prime} r^{\prime} q^{\prime} l^{\prime}\right),
\end{aligned}
$$

where $S(\{r\}, z)$ is as in Lemma 3.1 and $\xi(u)$ is the characteristic function of the interval $(x-y, x]$. Then, for $X^{\theta}(\log X)^{3 E+13} \leq Y \leq X(\log X)^{-3 E-11}$, we have

$$
R_{2}^{*}-R_{23}^{*}-\varrho_{1}-\varrho_{2}=y \lambda(x)+O_{\theta}\left(\frac{\delta Y}{\log X}\right)+\bar{O}_{\delta, E}\left(Y(\log X)^{-E}\right),
$$

where

$$
\begin{aligned}
& \varrho_{1}=\sum_{X^{\phi} \leq q \leq X^{(1-\theta) / 4}} \sum_{p<X^{(1-\theta+5 \Delta) / 2} q^{-1 / 2}} \sum_{r \leq X^{(1+\theta-5 \Delta) / 2} q^{-1 / 2}} S\left(\{r\}, X^{\phi}\right) \xi(p q r), \\
& \varrho_{2} \sum_{X^{\phi} \leq q \leq X^{(1-\theta) / 4}} \sum_{p>X^{(1+\theta-5 \Delta) / 2} q^{-1 / 2}} S\left(\{r\}, X^{\phi}\right) \xi(p q r),
\end{aligned}
$$

and $\lambda(x)$ is independent of $Y$.

Proof. By Buchstab's identity in the shape of (3.4),

$$
\begin{aligned}
& R_{2}^{*}=\sum_{X^{\phi} \leq q \leq X^{(1-\theta) / 4}} \sum_{p} \sum_{\substack{r \\
\frac{1}{16} X^{1-\theta+5} \leq q r^{2} \leq X^{1+\theta-5 \Delta}}} S\left(\{r\}, X^{\phi}\right) \xi(p q r) \\
& -\sum_{X^{\phi} \leq p^{\prime}<q<X^{(1-\theta) / 4}} \sum_{p} \sum_{\substack{r^{\prime} \\
\frac{1}{16} X^{1-\theta+5 \Delta} \leq q\left(p^{\prime} r^{\prime}\right)^{2} \leq X^{1+\theta-5 \Delta}}} S\left(\left\{r^{\prime}\right\}, p^{\prime}\right) \xi\left(p q p^{\prime} r^{\prime}\right) .
\end{aligned}
$$

The first sum on the right effectively contains as a subsum

$$
R_{21}^{*}=\sum_{X^{\phi} \leq q<X^{(1-\theta) / 4}} \sum_{\substack{p \\ X^{1-\theta+5 \Delta} \leq p^{2} q \leq X^{1+\theta-5 \Delta}}} S\left(\mathcal{A}_{p q}, X^{\phi}\right) ;
$$

this follows from the definition of $\xi(p q r)$, which certainly vanishes unless $(X / 4)^{2}<\left(p^{2} q\right)\left(q r^{2}\right)<X^{2}$ (note too that $p>X^{\phi}$ in $R_{21}^{*}$, for otherwise $p \leq q$ and $X^{1-\theta} \leq q^{3}$, contradicting $\left.q<X^{(1-\theta) / 4}\right)$. Similar considerations 
show that the remainder of the first sum (where either $p^{2} q<X^{1-\theta+5 \Delta}$ or $\left.p^{2} q>X^{1+\theta-5 \Delta}\right)$ is $\varrho_{1}+\varrho_{2}$.

In the other sum on the right $q\left(p^{\prime}\right)^{2}<X^{3(1-\theta) / 4}<X^{1-\theta+5 \Delta} / 16$, so that $r^{\prime} \neq 1$ and, in fact, $r^{\prime} \geq p^{\prime}$. Using the primality of $p$, we rewrite this sum as

$$
\begin{aligned}
\sum_{X^{\phi} \leq p^{\prime}<q<X^{(1-\theta) / 4}} \sum_{\substack{r^{\prime} \geq p^{\prime} \\
\frac{1}{16} X^{1-\theta+5 \Delta} \leq q\left(p^{\prime} r^{\prime}\right)^{2} \leq X^{1+\theta-5 \Delta}}} S\left(\left\{r^{\prime}\right\}, p^{\prime}\right) \\
\times \sum_{l} S\left(\{l\},\left(X /\left(q p^{\prime} r^{\prime}\right)\right)^{1 / 2}\right) \xi\left(q p^{\prime} r^{\prime} l\right)
\end{aligned}
$$

(note that $q p^{\prime} r^{\prime} \leq X^{(1+\theta-5 \Delta+(1-\theta) / 4) / 2}<X^{2 / 3}$ ). By applying Buchstab's identity (3.4) to each term of the inner sum here and collecting our results, we obtain

$$
R_{2}^{*}=R_{21}^{*}+\varrho_{1}+\varrho_{2}-R_{22}^{*}+R_{23}^{*},
$$

where

$$
R_{22}^{*}=\sum_{X^{\phi} \leq p^{\prime}<q<X^{(1-\theta) / 4}} \sum_{\substack{r^{\prime} \geq p^{\prime} \\ \frac{1}{16} X^{1-\theta+5 \Delta} \leq q\left(p^{\prime} r^{\prime}\right)^{2} \leq X^{1+\theta-5 \Delta}}} S\left(\left\{r^{\prime}\right\}, p^{\prime}\right) S\left(\mathcal{A}_{q p^{\prime} r^{\prime}}, X^{\phi}\right)
$$

and the other terms have already been defined. Baker and Harman [1], Section 6, had the same idea.

To complete the proof it suffices to obtain formulae for $R_{21}^{*}$ and $R_{22}^{*}$ similar to those given for $R_{11}$ and $R_{12}$ in the proof of Lemma 5.1. As this can be done in much the same way, we omit the details. In the applications of Proposition 3.1 the variable $q$ above will correspond to the variable $n$ of that proposition (for both $R_{21}^{*}$ and $R_{22}^{*}$ ). For $R_{21}^{*}, p$ will correspond to the variable $m$. For $R_{22}^{*}$ we put $p^{\prime} r^{\prime}=m$, so that the $a_{m}$ in Proposition 3.1 satisfy $0 \leq a_{m} \leq 1$.

LemmA 5.4. Let $\theta=1 / 14(\phi=12 / 77-\delta)$. Then, for $X^{1 / 14}(\log X)^{3 E+13}$ $\leq Y \leq X(\log X)^{-3 E-11}$,

$$
\begin{aligned}
R_{2} & =\sum_{X^{\phi} \leq q \leq X^{(1-\theta) / 4}} \sum_{\substack{p \\
\frac{1}{16} X^{1-\theta+5 \Delta} \leq p^{2} q \leq 16 X^{1+\theta-5 \Delta}}} S\left(\mathcal{A}_{p q}, q\right) \\
& \geq\left(a_{I I}-b_{I I}+O(\delta)\right) \frac{y}{\log X}+\bar{O}_{\delta, E}\left(Y(\log X)^{-E}\right),
\end{aligned}
$$

where

$$
a_{I I}=\int_{\beta=\phi}^{(1-\theta) / 4} \int_{\alpha=(1-\theta+5 \Delta-\beta) / 2}^{(1+\theta-5 \Delta-\beta) / 2} \omega\left(\frac{1-\alpha-\beta}{\beta}\right) \frac{d \alpha d \beta}{\alpha \beta^{2}}
$$


and

$$
b_{I I}=\underset{\substack{\phi<\varrho<\beta<(1-\theta) / 4 \\ \alpha+\beta>\varrho>\varrho \\ 1-\theta+5 \Delta<\beta+2 \varrho+2 \alpha<1+\theta-5 \Delta}}{\int} \int_{\sigma>\phi} \frac{d \alpha d \beta d \varrho d \sigma}{(1-\alpha-\beta-\varrho-\sigma) \alpha \beta \varrho \sigma} .
$$

Proof. Let $R_{2}^{*}, R_{23}^{*}, \varrho_{1}$ and $\varrho_{2}$ be as in Lemma 5.3. Since $R_{2} \geq R_{2}^{*}$, it will be enough to prove that the lower bound claimed for $R_{2}$ by the lemma is actually a lower bound for $R_{2}^{*}$. Therefore the lemma will follow from Lemma 5.3 , provided we can show that when $Y=X(\log X)^{-3 E-11}$ one has

$$
\begin{gathered}
\varrho_{1}, \varrho_{2}=O_{E}\left(Y(\log X)^{-2}\right), \\
R_{2}^{*} \geq\left(a_{I I}+O_{E}\left(\frac{1}{\log X}\right)\right) \frac{y}{\log X},
\end{gathered}
$$

and

$$
R_{23}^{*} \leq\left(b_{I I}+O_{E}\left(\frac{1}{\log X}\right)\right) \frac{y}{\log X},
$$

for $X / 2<x<X$; see the concluding part of the proof for Lemma 5.2.

To bound $\varrho_{1}$, we use

$$
\varrho_{1} \leq \sum_{X^{\phi} \leq q \leq X^{(1-\theta) / 4}} \sum_{\frac{1}{4} X^{(1-\theta+5 \Delta) / 2} q^{-1 / 2} \leq p \leq X^{(1-\theta+5 \Delta) / 2} q^{-1 / 2}} S\left(\mathcal{A}_{p q}, X^{\phi}\right) .
$$

Here $p q<X^{3(1-\theta) / 4}<X^{3 / 4}$, so that

$$
1<\frac{1}{4 \phi} \leq s=\frac{\log (X /(p q))}{\log \left(X^{\phi}\right)} \leq \frac{1}{\phi} .
$$

Hence Lemma 4.1 and (3.2) give us

$$
\begin{aligned}
\varrho_{1} & \ll_{E} \sum_{X^{\phi} \leq q \leq X^{(1-\theta) / 4}} \sum_{\frac{1}{4} X^{(1-\theta+5 \Delta) / 2} q^{-1 / 2} \leq p \leq X^{(1-\theta+5 \Delta) / 2} q^{-1 / 2}} \frac{y /(p q)}{\log X} \\
& \ll_{E} \frac{y}{\log X} \log \left(\frac{1-\theta}{4 \phi}\right) \frac{1}{\log \left(X^{3(1-\theta) / 8}\right)} \ll_{E} \frac{y}{(\log X)^{2}},
\end{aligned}
$$

as required. We omit the very similar treatment of $\varrho_{2}$.

For (5.8) we first use the inequality

$$
R_{2}^{*} \geq \sum_{X^{\phi} \leq q \leq X^{(1-\theta) / 4}} \sum_{\substack{p \\ X^{1-\theta+5 \Delta} \leq p^{2} q \leq X^{1+\theta-5 \Delta}}} S\left(\mathcal{A}_{p q}, q\right)=R_{2}^{* *} \text { (say). }
$$

It then suffices to prove (5.8) with $R_{2}^{* *}$ instead of $R_{2}^{*}$. This is accomplished by appeals to Lemmas 4.1 and 4.2 (see the treatment of $R_{1}$ in the proof for Lemma 5.2).

Turning lastly to (5.9) with $Y=X(\log X)^{-3 E-11}$, we begin by observing that in the sum $R_{23}^{*}$ both $r^{\prime}$ and $l^{\prime}$ are forced to be primes. Indeed, supposing 
$r^{\prime}$ to be composite, we find

$$
r^{\prime} \geq\left(p^{\prime}\right)^{2}, \quad q\left(p^{\prime}\right)^{6} \leq X^{1+\theta-5 \Delta} \quad \text { and } \quad 7 \phi<1+\theta ;
$$

and the last inequality contradicts our hypotheses. With $l^{\prime}$ we argue similarly, using the implicit inequality, $q\left(q^{\prime} l^{\prime}\right)^{2} \leq 16 X^{1+\theta-5 \Delta}$. By estimating the sum over $l^{\prime}$ with the prime number theorem (1.9), we find that

$$
\begin{aligned}
& R_{23}^{*} \leq \sum_{X^{\phi} \leq p^{\prime}<q \leq X^{(1-\theta) / 4}} \sum_{\substack{p \geq p^{\prime} \\
p q p^{\prime}\left(q^{\prime}\right)^{2}<X \\
\frac{1}{16} X^{1-\theta+5 \Delta} \leq q\left(p^{\prime} p\right)^{2} \leq X^{1+\theta-5 \Delta}}} \frac{y /\left(p^{\prime} q p q^{\prime}\right)}{\log \left(X /\left(p^{\prime} q p q^{\prime}\right)\right)} \\
& \times\left(1+O_{E}\left(\frac{1}{\log X}\right)\right) .
\end{aligned}
$$

To the right hand side here we apply Lemma 4.2 (or the prime number theorem), as it was applied to $R_{1}$ in the proof of Lemma 5.2. This yields (5.9), so completing the proof of the lemma.

With $\theta=1 / 14, \phi=12 / 77$ and $\Delta=0$, calculation yields

$$
c_{I I}=a_{I I}-b_{I I}=0.2046 \ldots-0.0219 \ldots=0.1827 \ldots
$$

Lemma 5.5. Let $h$ and $l$ be integers with $h, l \geq 2$ and $h \neq l$. Take $r$ to be the greatest integer strictly less than $\varrho=1 /(1-1 / h-1 / l)$ and put $\tau=(\varrho+r) / 2$. Suppose that $P \geq 2 Q, P Q^{2} \leq X / 4$,

$$
P \geq X^{\Delta+(1-\theta) / \min (h, \tau)}, \quad Q \geq X^{\Delta+(1-\theta) / \max (h, \tau)}
$$

and

$$
P Q \leq X^{1-\Delta-(1-\theta) / l} .
$$

Then, for $X^{\theta} \leq Y \leq X(\log X)^{-4-2 h l(E+3)}$,

$$
\begin{aligned}
R_{3}(P, Q) & =\sum_{P / 2<p \leq P} \sum_{Q / 2<q \leq Q} S\left(\mathcal{A}_{p q}, q\right) \\
& =\Lambda_{3}(P, Q) y+O_{h l, E, \delta}\left(Y(\log X)^{-4}\right)+\bar{O}_{h l, E}\left(Y(\log X)^{-E-2}\right),
\end{aligned}
$$

where

$$
\Lambda_{3}(P, Q)=\int_{u=P / 2}^{P} \int_{v=Q / 2}^{Q} \omega\left(\frac{\log (X /(u v))}{\log v}\right) \frac{d u d v}{u v(\log u)(\log v)^{2}} .
$$

Pr o of. We shall suppose that $X \geq 2^{12(h+6)}$, the lemma being otherwise trivial. The sum $R_{3}(P, Q)$ is of the same shape as the sum $R_{1}(P, Q)$ in the proof of Lemma 5.2. Hence we expect that

$$
R_{3}(P, Q)=\left(1+O_{h l, E}\left(\frac{1}{\log X}\right)\right) y \Lambda_{3}(P, Q),
$$


for $Y=X(\log X)^{-4-2 h l(E+3)}$ and $X / 2<x<X$. To verify that Lemmas 4.1 and 4.2 do give this, we need only observe

$$
\begin{gathered}
\frac{x-y}{p q}>\frac{X}{4 P Q}=\frac{X Q}{4 P Q^{2}} \geq Q \geq q, \\
s=\frac{\log (x /(p q))}{\log q} \leq \frac{\log X}{\log (Q / 2)}
\end{gathered}
$$

and

$$
Q>X^{1 /(2(h+\varrho))} \geq X^{1 /(2(h+6))} \geq 64 .
$$

As $\Lambda_{3}(P, Q)=O_{h}\left((\log X)^{-3}\right)$, we can complete the proof of the lemma by showing that, for all $Y$ in the assumed range,

$$
\frac{1}{y} R_{3}(P, Q)=\lambda(x)+O_{h l, \delta, E}\left((\log X)^{-4}\right)+\bar{O}_{h l, E}\left((\log X)^{-E-2}\right),
$$

with $\lambda(x)$ independent of $Y$.

As in the proof of Lemma 3.4,

$$
R_{3}(P, Q)=\Sigma^{ \pm} \pm \sigma^{ \pm}
$$

where $\sigma^{+} \geq 0, \sigma^{-} \geq 0$,

$$
\sigma^{+}+\sigma^{-}=\sum_{P / 2<p \leq P} \sum_{Q / 2<q \leq Q} \sum_{Q / 2 \leq p^{\prime}<Q} \sum_{m^{\prime}} S\left(\left\{m^{\prime}\right\}, p^{\prime}\right) \xi\left(p q p^{\prime} m^{\prime}\right)
$$

and

$$
\Sigma^{ \pm}=\sum_{P / 2<p \leq P} \sum_{Q / 2<q \leq Q} \sum_{m} S\left(\left\{m^{\prime}\right\}, Q^{ \pm}\right) \xi(p q m),
$$

with $Q^{+}=Q, Q^{-}=Q / 2$. To handle $\Sigma^{+}$and $\Sigma^{-}$, we take $A=h l(E+3)$, $T_{0}=\log ^{A} X, T=X^{1-\theta}(\log X)^{5}$ and

$$
\begin{aligned}
H^{ \pm}(t) & =\sum_{P / 2<p \leq P} \sum_{Q / 2<q \leq Q} \sum_{R / 16<m \leq R} S\left(\left\{m^{\prime}\right\}, Q^{ \pm}\right)(p q m)^{i t} \\
& =P(t) Q(t) R^{ \pm}(t) \quad \text { (say), }
\end{aligned}
$$

with $R=4 X /(P Q)$. Note that the condition $P Q^{2} \leq X / 4$ ensures that $R / 16 \geq Q$. As $p$ and $q$ here must be primes greater than $X^{1 /(4(h+6))}$, the case $r=1, \varepsilon=\Delta$ of Lemma 2.2 shows that

$$
\frac{1}{y} \Sigma^{ \pm}=\lambda^{ \pm}(x)+f^{ \pm}(x, y)+O_{h, \delta}\left((\log X)^{-4}\right),
$$

where $\lambda^{+}(x)$ and $\lambda^{-}(x)$ are independent of $Y$. Assuming (as may be done) that $X$ is sufficiently large in terms of $h l, E$ and $1 / \Delta$, one easily checks that the case $\varepsilon=1 /(4(h+6))$ of Lemma 2.7 applies; the required uniform bound 
(2.10) for $P(t)$ or $Q(t)$ being supplied by (1.7) with $B=h+6$. Since

$$
1-2\left(\frac{\varrho-r}{\varrho}\right) A \leq 1-\frac{2 A}{h l}=-2 E-5,
$$

we conclude, via Lemma 2.4, that

$$
f^{ \pm}(x, y)=\bar{O}_{h l, E}\left((\log X)^{-E-2}\right) .
$$

If $P Q^{3} \geq 16 X$, then one deduces from (5.12) that $\sigma^{+}=\sigma^{-}=0$. Hence (5.11), and the lemma, follow immediately from (5.13) and (5.14) in this case. For $P Q^{3}<16 X$, the proof can be completed, in much the same manner as the proof of Lemma 3.4, by means of (5.13), (5.14) and the upper bounds

$$
\begin{aligned}
\sigma^{+}+\sigma^{-} & \leq \sum_{P / 2<p \leq P} \sum_{Q / 2<q \leq Q} \sum_{Q / 2 \leq p^{\prime}<Q} S\left(\mathcal{A}_{p q p^{\prime}},\left(x /\left(p q p^{\prime}\right)\right)^{1 /(4(h+6))}\right) \\
& \ll_{h l, E} \sum_{P / 2<p \leq P} \sum_{Q / 2<q \leq Q} \sum_{Q / 2 \leq p^{\prime}<Q} \frac{y /\left(p q p^{\prime}\right)}{\log (Q / 32)} \\
& \ll_{h l, E} Y(\log X)^{-4},
\end{aligned}
$$

which hold at $Y=X(\log X)^{-4-2 h l(E+3)}$. These are obtained by the application of Lemma 4.1 and the prime number theorem.

Lemma 5.5 could be improved by observing that the Dirichlet polynomials $R^{ \pm}(t)$, from the proof, do satisfy a bound of the form (2.10) for $T_{0} \leq t \leq T$. We now give consideration to its applications, with the following choices of $h$ and $l$ :

$$
\begin{array}{ll}
\left(h_{1}, l_{1}\right)=(4,2), & \left(h_{2}, l_{2}\right)=(4,3), \\
\left(h_{3}, l_{3}\right)=(5,2), & \left(h_{4}, l_{4}\right)=(5,3), \\
\left(h_{5}, l_{5}\right)=(3,2), & \left(h_{6}, l_{6}\right)=(2,3) .
\end{array}
$$

To each pair $(h, l)$ corresponds a triple $(\alpha, \beta, \gamma)$, where $\alpha=(1-\theta) / \min (h, \tau)$, $\beta=(1-\theta) / \max (h, \tau), \gamma=1-(1-\theta) / l$ and $\tau$ is the arithmetic mean of $\varrho=1 /(1-1 / h-1 / l)$ and the greatest integer strictly less than $\varrho$ :

$$
\begin{aligned}
& \alpha_{1}=13 / 49, \quad \beta_{1}=13 / 56=\beta_{2}, \quad \alpha_{2}=65 / 154, \\
& \alpha_{3}=39 / 133, \quad \beta_{3}=13 / 70=\beta_{4}, \quad \alpha_{4}=13 / 29, \\
& \alpha_{5}=13 / 42, \quad \beta_{5}=13 / 77=\beta_{6}, \quad \alpha_{6}=13 / 28, \\
& \gamma_{1}=\gamma_{3}=\gamma_{5}=15 / 28 \quad \text { and } \quad \gamma_{2}=\gamma_{4}=\gamma_{6}=29 / 42 \text {. }
\end{aligned}
$$

For small $\Delta>0$ and $j=1, \ldots, 6$, we define a sum

$$
R_{3 j}=\sum_{\substack{X^{\alpha_{j}+\Delta} \leq p \leq X^{\alpha_{j+2}} \\ p q \leq X^{\gamma_{j}-\Delta}}} \sum_{X^{\beta_{j}+\Delta} \leq q<p} S\left(\mathcal{A}_{p q}, q\right),
$$


where $\alpha_{7}=\alpha_{8}=1 / 2$. Let $P$ and $Q$ be integer powers of 2 for which the sum $R_{3}(P, Q)$, in Lemma 5.5, is a non-trivial subsum of $R_{3 j}$. Then $P \geq 2 Q$ and

$$
P Q^{2}=(P Q)^{2} / P \leq 16 X^{2 \gamma-\alpha} \leq 16 X^{443 / 462}
$$

$\left(2 \gamma_{j}-\alpha_{j}\right.$ being maximal at $\left.j=2\right)$. Hence, provided that $X$ is sufficiently large, and that

$$
X^{1 / 14} \leq Y \leq X(\log X)^{-90-30 E},
$$

Lemma 5.5 will yield the stated formula:

$$
R_{3}(P, Q)=\Lambda_{3}(P, Q) y+O_{E, \delta}\left(Y(\log X)^{-4}\right)+\bar{O}_{E}\left(Y(\log X)^{-E-2}\right) .
$$

Since $\Lambda_{3}(P, Q)=O\left((\log X)^{-3}\right)$, it is not hard to show that

$$
\sum_{P, Q} \Lambda_{3}(P, Q)=c_{3 j}(\log X)^{-1}+O\left((\log X)(\log X)^{-3}\right),
$$

where the summation is taken over all the values that can be taken (above) by $P$ and $Q$, and

$$
c_{3 j}=\int_{\alpha=\alpha_{j}+\Delta}^{\alpha_{j+2}} \int_{\substack{\alpha=\beta_{j}+\Delta \\ \alpha+\beta \leq \gamma_{j}-\Delta}}^{\alpha} \omega\left(\frac{1-\alpha-\beta}{\beta}\right) \frac{d \alpha d \beta}{\alpha \beta^{2}} .
$$

As there are only $O\left((\log X)^{2}\right)$ choices for $P$ and $Q$, we conclude (via (1.4)) that

$$
\begin{aligned}
R_{3 j} & \geq c_{3 j} \frac{y}{\log X}+O_{E, \delta}\left(Y(\log X)^{-2}\right)+\bar{O}_{E}\left(Y(\log X)^{-E}\right) \\
& =\left(c_{3 j}+O(\delta)\right) \frac{y}{\log X}+\bar{O}_{E, \delta}\left(Y(\log X)^{-E}\right),
\end{aligned}
$$

for $X$ and $Y$ as in (5.18).

With (5.17) we showed in effect that $\alpha+2 \beta \leq 1$ in the integral (5.19). As $\beta>0$, this implies: $s=(1-\alpha-\beta) / \beta \geq \beta / \beta=1$. One easily verifies that $2 \alpha_{j} \geq \gamma_{j}$ for $j=2, \ldots, 6$, so the condition $\beta \leq \alpha$ (or $\alpha+\beta \leq 2 \alpha$ ) is redundant in (5.19) unless $j=1$. When $j=5$ the condition $\alpha \leq \alpha_{7}=1 / 2$ is redundant. We calculate that when $\Delta=0$,

$$
\begin{aligned}
c_{i} & =c_{31}+c_{33}+c_{35} \\
& =0.0211 \ldots+0.0330 \ldots+0.0806 \ldots=0.1348 \ldots, \\
c_{i i} & =c_{32}+c_{34}+c_{36} \\
& =0.0177 \ldots+0.0243 \ldots+0.0473 \ldots=0.0894 \ldots
\end{aligned}
$$

6. Summary of results and deduction of Theorem 1. Throughout this last section $\theta=1 / 14$ and $\phi=12 / 77-\delta$, where $\delta=\Delta^{2}$ and $0<\Delta<$ 
$10^{-3}$. It will be enough to prove Theorem 1 for $Y$ satisfying

$$
X^{1 / 14}(\log X)^{3 E+13} \leq Y \leq X(\log X)^{-90-30 E} ;
$$

for larger $Y$ the asymptotic formula (1.1) holds, by virtue of the prime number theorem (1.9).

Before we can use the results of Sections 4 and 5 , we need to verify that the sums $R_{1}, R_{2}, R_{31}, \ldots, R_{36}$ (from Lemmas 5.1 and 5.4, (5.15) and (5.16)) are subsums of $R$ (from (1.8)) and that they do satisfy a condition of pairwise disjointness.

For the first half of this, it suffices to show that each of the conditions, $q \geq X^{\phi}, q<p$ and $p \leq X^{1 / 2}$, is present (explicitly or implicitly) in all of the sums. This is easily accomplished for $R_{1}$, where $X^{\phi} \leq q<p$ is given and one can deduce that $p^{2} \leq X^{1+\theta-5 \Delta-3 \phi} \leq X$. For $R_{2}$ we have: $X^{\phi} \leq q$ (explicit), $p^{2} \leq 16 X^{1+\theta-5 \Delta-\phi}<16 X^{1-1 / 14}<X$ and $p^{2} / q^{2} \geq \frac{1}{16} X^{(1-\bar{\theta}) / 4}>1$. The sums $R_{31}, \ldots, R_{36}$ all have $q<p$, so it is enough to note that $\beta_{1}, \ldots, \beta_{6} \geq$ $13 / 77>\phi$ and $\alpha_{3}, \ldots, \alpha_{8} \leq 1 / 2$ (see (5.15) and (5.16)).

For the second half (pairwise disjointness) we consider the values of $2 \alpha+\beta$ at points $(\alpha, \beta)=(\log p / \log X, \log q / \log X)$ corresponding to terms of the sums in question. For $R_{1}, 2 \alpha+\beta \leq 1+\theta-5 \Delta-2 \phi<15 / 14-24 / 77=$ $117 / 154$. For $R_{31}, R_{33}$, or $R_{35}, 2 \alpha+\beta \geq 2 \alpha_{1}+\beta_{1}=26 / 49+13 / 56=$ $299 / 392>117 / 154$ and $2 \alpha+\beta \leq 2 \gamma_{5}-\beta_{5}=15 / 14-13 / 77=139 / 154$. For $R_{2}, 15 / 14 \geq 2 \alpha+\beta \geq 13 / 14>139 / 154$. Finally, for $R_{32}, R_{34}$, or $R_{36}$, $2 \alpha+\beta \geq 2 \alpha_{2}+\beta_{2}=65 / 77+13 / 56=663 / 616>15 / 14$. We conclude that if two of the sums $R_{1}, R_{2}, R_{31}, \ldots, R_{36}$ have a term in common, then they are $R_{3 i}$ and $R_{3 j}$, for some $i, j$ with $i \equiv j(\bmod 2)$. The condition $X^{\alpha_{j}}<p \leq X^{\alpha_{j+2}}$ in (5.16) shows that there are no such pairs.

Since $R_{1}, R_{2}, R_{31}, \ldots, R_{36}$ are pairwise disjoint subsums of $R$, their sum is a subsum of $R$. As all terms are positive, or zero, it follows that

$$
R \geq R_{1}+R_{2}+R_{31}+\ldots+R_{36}=R^{\prime} \quad \text { (say). }
$$

Hence, Lemmas 5.2 and 5.4 and (5.20) together imply that

$$
R \geq R^{\prime} \geq\left(c^{\prime}+O(\delta)\right) \frac{y}{\log X}+\bar{O}_{\delta, E}\left(Y(\log X)^{-E}\right),
$$

where

$$
\begin{aligned}
c^{\prime} & =c_{I}+c_{I I}+c_{i}+c_{i i} \\
& =0.3567 \ldots+0.1827 \ldots+0.1348 \ldots+0.0894 \ldots+O(\Delta) \\
& =0.7636 \ldots+O(\Delta)
\end{aligned}
$$

(see (5.7), (5.10), (5.21) and the definitions of $a_{I}, b_{I}, a_{I I}, b_{I I}, c_{31}, \ldots, c_{36}$ given in Lemma 5.2, Lemma 5.4 and (5.19)). By the above, (4.5)-(4.7),

$$
S \geq \frac{c y}{\log X}+\bar{O}_{\Delta, E}\left(Y(\log X)^{-E}\right),
$$


where $S=\pi(x)-\pi(x-y)$, from (1.8), and

$$
\begin{aligned}
c=c_{1}-c_{2}+c^{\prime}+O(\delta) & =1-c_{3}+c^{\prime}+O(\delta) \\
& \geq 1-1.5966+0.7636+O(\Delta) .
\end{aligned}
$$

Since $0.7636-0.5966=0.167$, there is a numerical constant $\Delta_{0}>0$ which is sufficiently small that Theorem 1 follows on taking $\Delta=\Delta_{0}$.

\section{References}

[1] R. C. Baker and G. Harman, The difference between consecutive primes, preprint.

[2] A. Buchstab, Asymptotic estimates of a general number-theoretic function, Mat. Sb. (N.S.) (2) 44 (1937), 1239-1246 (in Russian with a German summary).

[3] H. Davenport, Multiplicative Number Theory, Springer, 1980.

[4] J.-M. Deshouillers and H. Iwaniec, Kloosterman sums and Fourier coefficients of cusp forms, Invent. Math. 70 (1982), 219-288.

[5] - - - Power mean values of the Riemann zeta-function, Mathematika 29 (1982), $202-212$.

[6] G. H. Hardy and E. M. Wright, An Introduction to the Theory of Numbers, Clarendon Press, Oxford, 1954.

[7] G. Harman, Almost-primes in short intervals, Math. Ann. 258 (1981), 107-112.

[8] -, Primes in short intervals, Math. Z. 180 (1982), 335-348.

[9] - On the distribution of ap modulo one, J. London Math. Soc. (2) 27 (1983), 9-18.

[10] - On the distribution of ap modulo one II, preprint.

[11] D. R. Heath-Brown, Gaps between primes and the pair correlation of zeros of the zeta-function, Acta Arith. 41 (1982), 85-99.

[12] —, Finding primes by sieve methods, Proc. 1982 ICM, Warsaw, 1983, PWN, Vol. 1, Warszawa, 1984, 487-492.

[13] D. R. Heath-Brown and H. Iwaniec, On the difference between consecutive primes, Invent. Math. 55 (1979), 49-69.

[14] M. N. Huxley, On the difference between consecutive primes, Invent. Math. 15 (1972), 164-170.

[15] H. Iwaniec, Rosser's sieve, Acta Arith. 36 (1980), 171-202.

[16] —, A new form of the error term in the linear sieve, ibid. 37 (1980), 307-320.

[17] H. Iwaniec and M. Jutila, Primes in short intervals, Ark. Mat. 17 (1979), 167176.

[18] H. Iwaniec and J. Pintz, Primes in short intervals, Monatsh. Math. 98 (1984), $115-143$

[19] C. Jia, On the Goldbach numbers in the short interval, Science in China, to appear

[20] - On the difference between consecutive primes, ibid., to appear.

[21] H. Li, Primes in short intervals, unpublished manuscript.

[22] - Primes in short intervals, preprint.

[23] S. Lou and Q. Yao, A Chebychev's type of prime number theorem in a short interval - II, Hardy-Ramanujan J. 15 (1992), 1-33.

[24] H. Mikaw a, Almost-primes in arithmetic progressions and short intervals, Tsukuba J. Math. (2) 13 (1989), 387-401.

[25] H. L. Montgomery, Topics in Multiplicative Number Theory, Lecture Notes in Math. 227, Springer, 1971. 
[26] H. L. Montgomery and R. C. Vaughan, Hilbert's inequality, J. London Math. Soc. (2) 8 (1974), 73-82.

[27] —, - The exceptional set in Goldbach's problem, Acta Arith. 27 (1975), 353-370.

[28] Y. Motohashi, A note on almost-primes in short intervals, Proc. Japan Acad. Ser. A Math. Sci. 55 (1979), 225-226.

[29] C. J. Mozzochi, On the difference between consecutive primes, J. Number Theory 24 (1986), 181-187.

[30] A. Perelli and J. Pintz, On the exceptional set for Goldbach's Problem in short intervals, J. London Math. Soc. (2) 47 (1993), 41-49.

[31] A. Selberg, On the normal density of primes in short intervals, and the difference between consecutive primes, Arch. Math. Naturvid. 47 (1943), 87-105.

[32] P. Shiu, A Brun-Titchmarsh theorem for multiplicative functions, J. Reine Angew. Math. 313 (1980), 161-170.

[33] E. C. Titchmarsh, The Theory of the Riemann Zeta-Function, Clarendon Press, Oxford, 1986.

[34] N. W at t, Kloosterman sums and a mean value for Dirichlet polynomials, J. Number Theory, to appear.

[35] D. Wolke, Fast-Primzahlen in kurzen Intervallen, Math. Ann. 224 (1979), 233-242.

DEPARTMENT OF MATHEMATICS

UNIVERSITY OF NOTTINGHAM

UNIVERSITY PARK

NOTTINGHAM NG7 2RD, UNITED KINGDOM

E-mail: NIGEL.WATT@MATHS.NOTTINGHAM.AC.UK

Received on 17.6.1994

and in revised form on 12.12.1994 